\title{
Structural and Redox Variations in Technetium Complexes Supported by $m$-Terphenyl Isocyanides
}

Guilhem Claude ${ }^{\dagger}$ Federico Salsi,${ }^{\dagger}$ Adelheid Hagenbach,${ }^{\dagger}$ Milan Gembicky, ${ }^{\star}$ Michael Neville, ${ }^{\ddagger}$ Chinglin Chan, ${ }^{\ddagger}$ Joshua S. Figueroa, ${ }^{\ddagger *}$ and Ulrich Abram ${ }^{\dagger *}$

$\dagger \quad$ Freie Universität Berlin, Institute of Chemistry and Biochemistry, Fabeckstr. 34/36, D14195 Berlin, Germany

\$ Department of Chemistry and Biochemistry, University of California, San Diego, $9500 \mathrm{G}$ ilman Drive, Mail Code 0358, La Jolla, CA 92093, USA 


\section{Crystallographic data}

Table S1. Crystal data and structure determinations parameters

\begin{tabular}{|c|c|c|c|}
\hline & $\begin{array}{l}{\left[\mathrm{Tc}(\mathrm{COO})_{3} \mathrm{Cl}-\right.} \\
\left.(\mathrm{CNArDipp2})_{2}\right]\end{array}$ & $\begin{array}{l}{\left[\mathrm{Tc}(\mathrm{NO}) \mathrm{Cl}_{2}\left(\mathrm{PPh}_{3}\right)-\right.} \\
\left.(\mathrm{CNArDipp2})_{2}\right]\end{array}$ & $\begin{array}{l}{\left[\mathrm{Tc}(\mathrm{PPhMe})_{2} \mathrm{Cl}_{3-}\right.} \\
(\mathrm{CNArDipp2})]\end{array}$ \\
\hline Formula & $\mathrm{C}_{65} \mathrm{H}_{74} \mathrm{CIN}_{2} \mathrm{O}_{3} \mathrm{Tc}$ & $\mathrm{C}_{80} \mathrm{H}_{89} \mathrm{Cl}_{2} \mathrm{~N}_{3} \mathrm{OPTc}$ & $\mathrm{C}_{47} \mathrm{H}_{59} \mathrm{Cl}_{3} \mathrm{NO} \mathrm{P}_{2} \mathrm{Tc}$ \\
\hline Mw & 1064.71 & 1308.41 & 904.24 \\
\hline Crystal system & Monoclinic & Monoclinic & Triclinic \\
\hline $\mathrm{a} / \AA ̊$ & $24.069(2)$ & $22.217(2)$ & $12.099(2)$ \\
\hline$b / \AA ̊$ & 23.959(2) & $21.732(2)$ & $12.807(3)$ \\
\hline$c / \AA ̊$ & $21.170(2)$ & $16.845(1)$ & $16.575(3)$ \\
\hline$\alpha /^{\circ}$ & 90 & 90 & $77.22(3)$ \\
\hline$\beta /^{\circ}$ & $108.44(1)$ & $112.079(3)$ & $75.96(3)$ \\
\hline $\mathrm{Y} /^{\circ}$ & 90 & 90 & $66.99(3)$ \\
\hline $\mathrm{V} / \AA^{3}$ & $11582.4(14)$ & $7536.7(1)$ & $2270.4(10)$ \\
\hline Space group & $\mathrm{C} 2 / \mathrm{c}$ & $P 21 / c$ & $\overline{\mathrm{P}} 1$ \\
\hline Z & 8 & 4 & 2 \\
\hline$\rho_{\text {calc }} /\left(\mathrm{g} \mathrm{cm}^{-3}\right)$ & 1.221 & 1.153 & 1.323 \\
\hline$\mu / \mathrm{mm}^{-1}$ & 0.340 & 0.327 & 0.596 \\
\hline No. reflec & 208596 & 105365 & 20249 \\
\hline No. Indepen. & 12809 & 16173 & 9562 \\
\hline $\mathrm{R}_{\text {int }}$ & 0.0322 & 0.0459 & 0.0684 \\
\hline No. Param. & 637 & 792 & 487 \\
\hline$R_{1} / \mathrm{w} R_{2}$ & $0.0323 / 0.0773$ & $0.0464 / 0.1068$ & $0.0517 / 0.1043$ \\
\hline GOF & 1.031 & 1.024 & 0.933 \\
\hline CCDC & 1989548 & 1989549 & 1989550 \\
\hline
\end{tabular}


Table S1 (Continued). Crystal data and structure determinations parameters

\begin{tabular}{|c|c|c|c|}
\hline & $\begin{array}{l}\text { trans- } \\
{\left[\mathrm{TCNCl}_{2}\left(\mathrm{CNArDipp}_{2}\right)_{2}\right]}\end{array}$ & $\begin{array}{l}\left.\text { trans-[Tc(NPh } \mathrm{Cl}_{3}\left(\mathrm{CNAr}^{\mathrm{Dipp} 2}\right)_{2}\right] \cdot \\
\text { toluene }\end{array}$ & $\begin{array}{l}\text { trans-[Tc(NPh)Br }{ }_{3}- \\
\left.(\mathrm{CNArDipp2})_{2}\right] \cdot \text { toluene }\end{array}$ \\
\hline Formula & $\mathrm{C}_{62} \mathrm{H}_{74} \mathrm{Cl}_{2} \mathrm{~N}_{3} \mathrm{Tc}$ & $\mathrm{C}_{75} \mathrm{H}_{87} \mathrm{Cl}_{3} \mathrm{~N}_{3} \mathrm{Tc}$ & $\mathrm{C}_{75} \mathrm{H}_{87} \mathrm{Br}_{3} \mathrm{~N}_{3} \mathrm{Tc}$ \\
\hline Mw & 1030.14 & 1234.82 & 1368.20 \\
\hline Crystal system & Triclinic & Monoclinic & Monoclinic \\
\hline $\mathrm{a} / \AA \AA$ & $16.717(1)$ & $17.496(3)$ & $17.669(1)$ \\
\hline $\mathrm{b} / \AA ̊ \AA$ & $17.096(1)$ & $15.192(5)$ & $15.223(1)$ \\
\hline$c / \AA ̊$ & 21.109(2) & $26.200(6)$ & $26.232(2)$ \\
\hline$\alpha /^{\circ}$ & $77.332(2)$ & 90 & 90 \\
\hline$\beta /^{\circ}$ & $75.787(2)$ & $99.997(6)$ & $99.515(3)$ \\
\hline $\mathrm{y} /{ }^{\circ}$ & $89.879(3)$ & 90 & 90 \\
\hline$V / \AA^{3}$ & $5697.1(8)$ & $6858(3)$ & $6959(1)$ \\
\hline Space group & $\overline{\mathrm{P}} 1$ & $P 2_{1} / c$ & $P 2_{1} / c$ \\
\hline Z & 4 & 4 & 4 \\
\hline$\rho_{\text {calc }} /\left(\mathrm{g} \mathrm{cm}^{-3}\right)$ & 1.201 & 1.196 & 1.306 \\
\hline$\mu / \mathrm{mm}^{-1}$ & 0.386 & 0.369 & 1.971 \\
\hline No. reflec & 71434 & 241318 & 128572 \\
\hline No. Indepen. & 71434 & 16339 & 14298 \\
\hline $\mathrm{R}_{\text {int }}$ & - & 0.0420 & 0.0914 \\
\hline No. Param. & 1289 & 756 & 756 \\
\hline$R_{1} / \mathrm{w} R_{2}$ & $0.0619 / 0.1229$ & $0.0272 / 0.616$ & $0.0373 / 0.0604$ \\
\hline GOF & 1.057 & 1.082 & 1.023 \\
\hline CCDC & 1989551 & 1989552 & 1989553 \\
\hline
\end{tabular}


Table S1 (Continued). Crystal data and structure determinations parameters

\begin{tabular}{|c|c|c|c|}
\hline & $\begin{array}{l}{\left[\mathrm{Tc}\left(\mathrm{PPhMe}_{2}\right)_{2-}\right.} \\
\left.\mathrm{Cl}_{3}\left(\mathrm{CNAr}^{\mathrm{Mes} 2}\right)\right]\end{array}$ & $\begin{array}{l}\text { cis-[TcNCl }\left(\mathrm{CNArMes}^{2}\right)- \\
(\mathrm{MeOH})]\end{array}$ & $\begin{array}{l}\text { cis-[TcNCl }\left(\mathrm{CNArMes}^{2}\right)_{2}- \\
\left.\left(\mathrm{H}_{2} \mathrm{O}\right)\right]\end{array}$ \\
\hline Formula & $\mathrm{C}_{41} \mathrm{H}_{47} \mathrm{Cl}_{3} \mathrm{NP}_{2} \mathrm{Tc}$ & $\mathrm{C}_{51} \mathrm{H}_{54} \mathrm{Cl}_{2} \mathrm{~N}_{3} \mathrm{OTc}$ & $\mathrm{C}_{50} \mathrm{H}_{52} \mathrm{Cl}_{2} \mathrm{~N}_{3} \mathrm{OTc}$ \\
\hline Mw & 820.08 & 893.87 & 879.84 \\
\hline Crystal system & Monoclinic & Monoclinic & Monoclinic \\
\hline $\mathrm{a} / \AA ̊$ & $11.099(2)$ & $13.567(1)$ & $13.535(1)$ \\
\hline $\mathrm{b} / \AA ̊ \AA$ & $19.552(4)$ & $18.154(2)$ & $17.979(2)$ \\
\hline$c / \AA ̊$ & $18.557(4)$ & $19.494(2)$ & $19.541(2)$ \\
\hline$\alpha /^{\circ}$ & 90 & 90 & 90 \\
\hline$\beta /^{\circ}$ & $92.24(3)$ & $104.260(4)$ & $103.991(3)$ \\
\hline$y /{ }^{\circ}$ & 90 & 90 & 90 \\
\hline $\mathrm{V} / \AA^{3}$ & $4024(1)$ & $4653.2(8)$ & $4614.0(7)$ \\
\hline Space group & $P 2_{1} / n$ & $P 2_{1} / n$ & $\mathrm{P} 22_{1} / \mathrm{n}$ \\
\hline Z & 4 & 4 & 4 \\
\hline$\rho_{\text {calc }} /\left(\mathrm{g} \mathrm{cm}^{-3}\right)$ & 1.354 & 1.276 & 1.267 \\
\hline$\mu / \mathrm{mm}^{-1}$ & 0.666 & 0.463 & 0.466 \\
\hline No. reflec & 23021 & 84517 & 130699 \\
\hline No. Indepen. & 8481 & 10316 & 10228 \\
\hline $\mathrm{R}_{\text {int }}$ & 0.0500 & 0.0683 & 0.0616 \\
\hline No. Param. & 383 & 544 & 548 \\
\hline$R_{1} / \mathrm{w} R_{2}$ & $0.0477 / 0.1018$ & $0.0900 / 0.2016$ & $0.0852 / 0.1874$ \\
\hline GOF & 0.996 & 1.356 & 1.293 \\
\hline CCDC & 1989554 & 1989555 & 1989556 \\
\hline
\end{tabular}


Figure S1. Ellipsoid representations (50\% probability) of trans-[Tc(CO) $\left.{ }_{3} \mathrm{Cl}\left(\mathrm{CNAr}^{\mathrm{Dipp} 2}\right)_{2}\right]$.

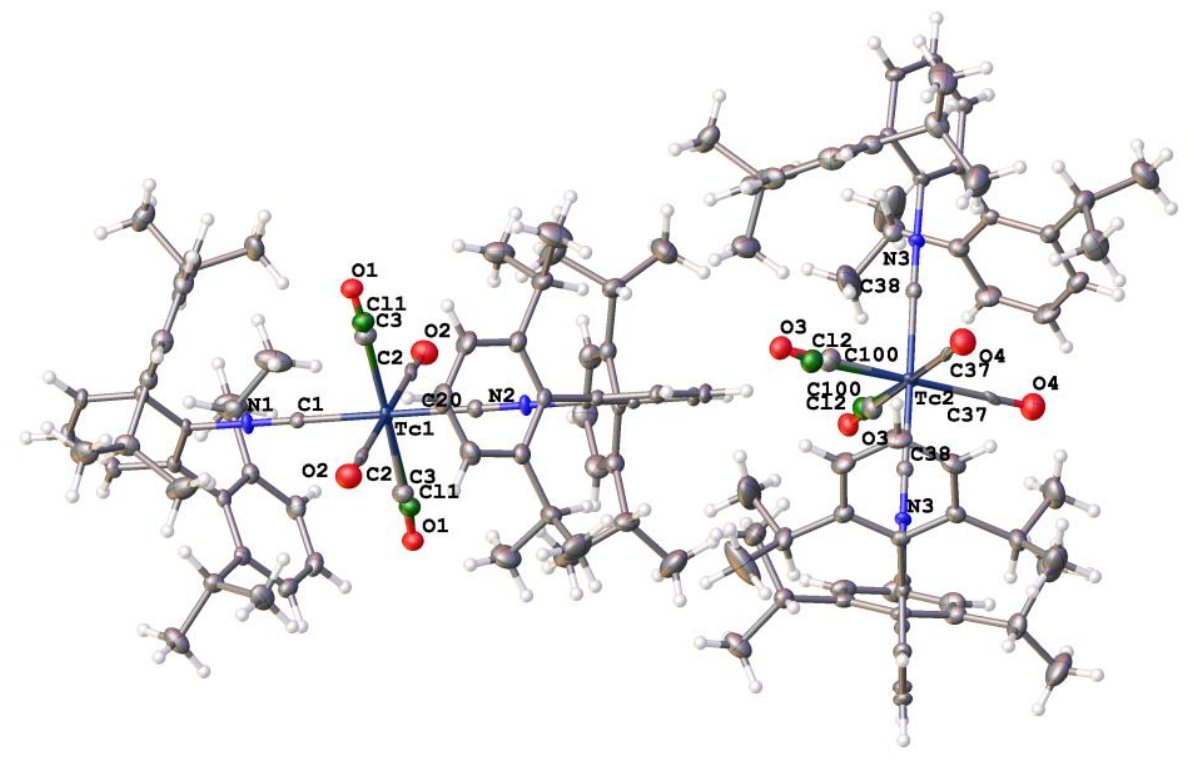

Table S2. Selected bond lengths $(\AA)$ and bond angles $\left(^{\circ}\right)$ in trans $-\left[\mathrm{Tc}(\mathrm{CO})_{3} \mathrm{Cl}(\mathrm{CNArDipp} 2)_{2}\right]$.

\begin{tabular}{llll}
\hline Bond lengths & & & \\
\hline Tc1-C1 & $2.049(2)$ & C20-N2 & $1.151(3)$ \\
Tc1-C20 & $2.057(2)$ & Tc2-C38 & $2.052(2)$ \\
Tc1-C2 & $2.066(2)$ & Tc2-C100 & $1.979(6)$ \\
Tc1-C3 & $1.970(5)$ & Tc2-C37 & $2.058(2)$ \\
Tc1-Cl1 & $2.334(9)$ & Tc2-Cl2 & $2.396(1)$ \\
C1-N1 & $1.154(3)$ & C38-N3 & $1.154(2)$ \\
\hline Bond angles & & & \\
\hline C1-Tc1-C20 & $168.23(2)$ & C38-Tc2-C38' & $179.43(8)$ \\
C1-Tc1-C2 & $89.93(4)$ & C38-Tc2-C37 & $89.97(6)$ \\
C1-Tc1-C2' & $89.93(4)$ & C38-Tc2-C37' & $90.41(6)$ \\
C1-Tc1-C3 & $88.6(2)$ & C38-Tc2-C100 & $87.8(2)$ \\
C1-Tc1-Cl1 & $90.08(4)$ & C38-Tc2-C100' & $87.8(2)$ \\
C20-Tc1-C2 & $90.07(4)$ & C38-Tc2-Cl2 & $90.51(5)$ \\
C20-Tc1-C2' & $90.07(4)$ & C37-Tc2-C37’ & $96.62(6)$ \\
C20-Tc1-C3 & $91.4(2)$ & C37-Tc2-C100 & $177.2(2)$ \\
C20-Tc1-Cl1 & $89.92(4)$ & C37-Tc2-C100' & $177.2(2)$ \\
C2-Tc1-C2 & $179.86(8)$ & C37-Tc2-Cl2 & $177.51(5)$ \\
C2-Tc1-C3 & $87.7(2)$ & C100-Tc2-C110' & $93.3(5)$ \\
C2-Tc1-Cl1 & $85.68(5)$ & C100'-Tc2-Cl2 & $92.5(2)$ \\
C3-Tc1-Cl1 & $177.6(2)$ & & \\
\hline
\end{tabular}


Figure S2. Ellipsoid representation (50\% probability) of trans-[Tc(NO)Cl $\left.2\left(\mathrm{PPh}_{3}\right)\left(\mathrm{CNAr}^{\mathrm{Dipp} 2}\right)_{2}\right]$ Hydrogen atoms are omitted for clarity.

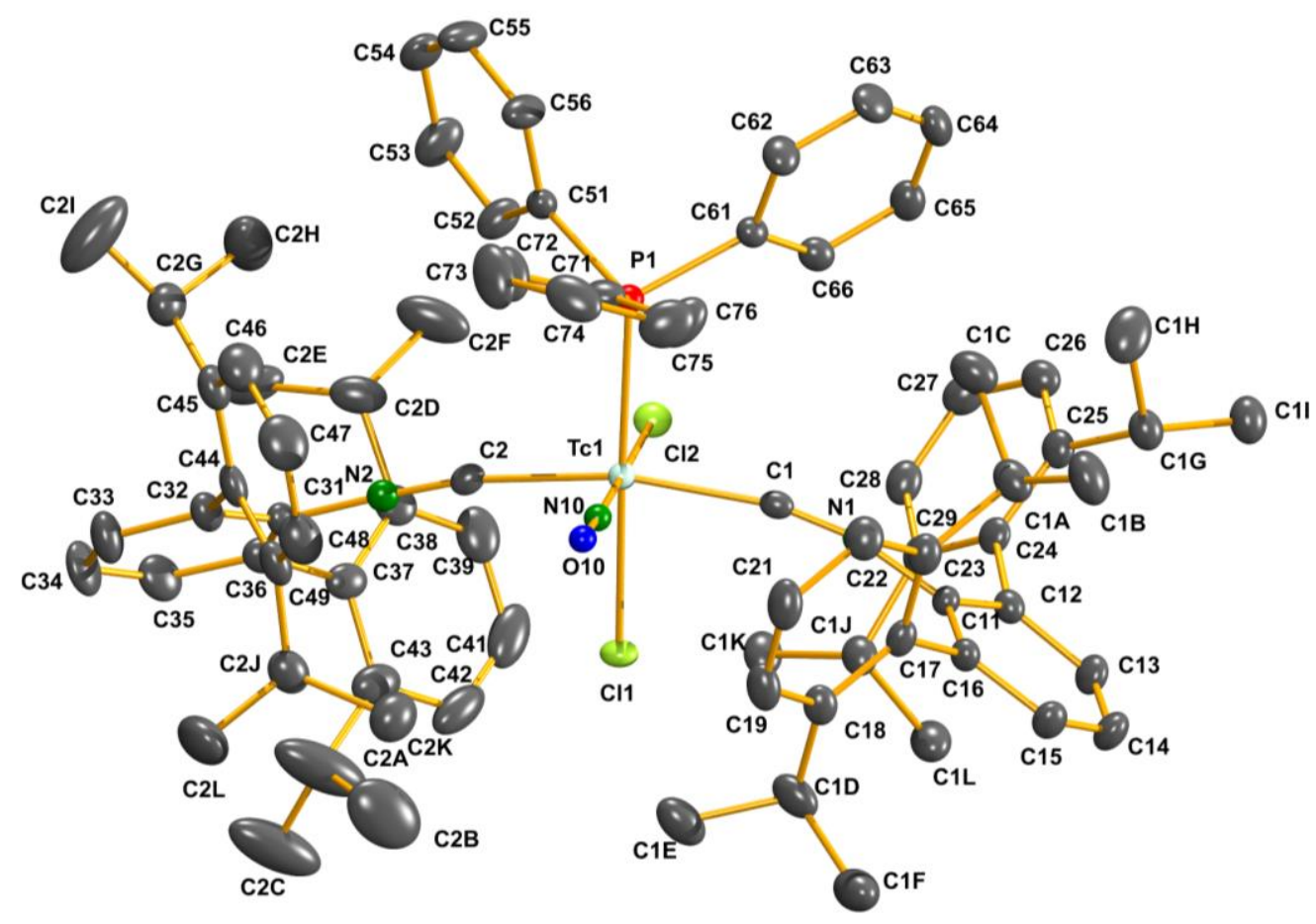

Table S3. Selected bond lengths $(\AA)$ and angles $\left(^{\circ}\right)$ in trans- $\left[\mathrm{Tc}(\mathrm{NO}) \mathrm{Cl}_{2}\left(\mathrm{PPh}_{3}\right)\left(\mathrm{CNAr}{ }^{\mathrm{Dipp} 2}\right)_{2}\right]$

\begin{tabular}{|c|c|c|c|}
\hline \multicolumn{4}{|l|}{ Bond lengths } \\
\hline Tc1-P1 & $2.4178(6)$ & $\mathrm{N} 10-\mathrm{O} 10$ & $1.088(2)$ \\
\hline Tc1-Cl1 & $2.4581(6)$ & Tc1-C1 & $2.064(2)$ \\
\hline Tc1-Cl2 & $2.4034(7)$ & Tc1-C2 & $2.064(2)$ \\
\hline Tc1-N10 & $1.8320(1)$ & N1-C11 & $1.401(3)$ \\
\hline C1-N1 & $1.157(3)$ & C2-N2 & $1.161(3)$ \\
\hline \multicolumn{4}{|l|}{ Bond angles } \\
\hline N10-Tc1-P1 & $168.23(2)$ & C1-Tc1-Cl1 & $82.62(6)$ \\
\hline O10-N10-Tc1 & $177.4(2)$ & $\mathrm{C} 1-\mathrm{Tc} 1-\mathrm{Cl} 2$ & $88.47(6)$ \\
\hline N10-Tc1-Cl1 & $91.76(6)$ & $\mathrm{C} 2-\mathrm{Tc} 1-\mathrm{Cl} 1$ & $83.74(7)$ \\
\hline N10-Tc1-Cl2 & $179.49(6)$ & $\mathrm{C} 1-\mathrm{Tc} 1-\mathrm{P} 1$ & $96.35(6)$ \\
\hline $\mathrm{Cl} 2-\mathrm{Tc} 1-\mathrm{Cl} 1$ & $88.68(2)$ & N1-C1-Tc1 & $167.7(2)$ \\
\hline Cl2-Tc1-P1 & $88.85(2)$ & N2-C2-Tc1 & $170.5(2)$ \\
\hline P1-Tc1-Cl1 & $177.35(2)$ & C11-C1-N1 & $167.2(2)$ \\
\hline C1-Tc1-C2 & $165.97(9)$ & C31-C2-N2 & $177.7(2)$ \\
\hline
\end{tabular}


Figure S3. Ellipsoid representation (50\% probability) of mer-[Tc( $\left.\left.\mathrm{PPhMe}_{2}\right)_{2} \mathrm{Cl}_{3}\left(\mathrm{CNAr}{ }^{\mathrm{Dipp} 2}\right)\right]$ Hydrogen atoms are omitted for clarity.

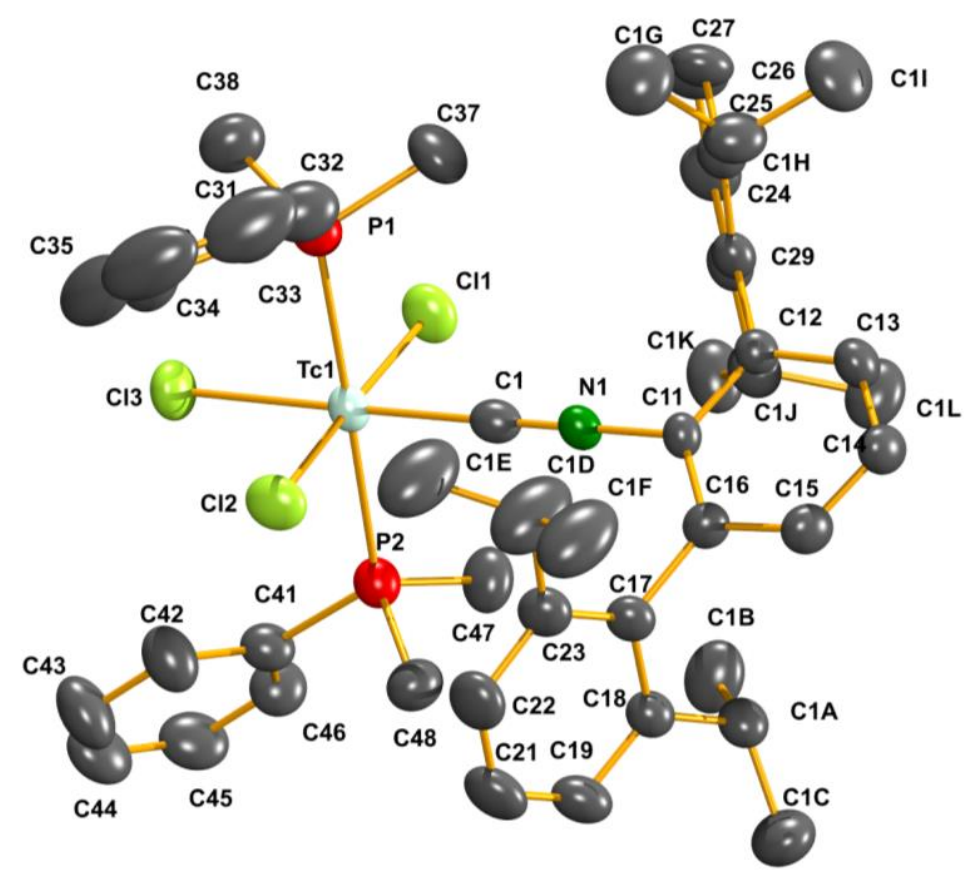

Table S4. Selected bond lengths $(\AA)$ and bond angles $\left(^{\circ}\right)$ in mer-[Tc $\left.\left(\mathrm{PPhMe}_{2}\right)_{2} \mathrm{Cl}_{3}\left(\mathrm{CNAr}^{\mathrm{Dipp} 2}\right)\right]$

\begin{tabular}{llll}
\hline Bond lengths & & & \\
\hline Tc1-C1 & $2.034(5)$ & Tc1-Cl1 & $2.333(2)$ \\
Tc1-P1 & $2.464(2)$ & Tc1-Cl2 & $2.322(2)$ \\
Tc1-P2 & $2.466(2)$ & Tc1-Cl3 & $2.425(1)$ \\
C1-N1 & $1.172(5)$ & N1-C1 & $1.415(5)$ \\
\hline Bond angles & & & \\
\hline Cl1-Tc1-Cl3 & $91.76(5)$ & Cl2-Tc1-Cl1 & $177.42(5)$ \\
Cl1-Tc1-P1 & $85.70(6)$ & Cl2-Tc1-Cl3 & $90.79(6)$ \\
Cl2-Tc1-P2 & $87.25(6)$ & Cl2-Tc1-P1 & $94.06(6)$ \\
N1-C1-Tc1 & $179.6(4)$ & Cl3-Tc1-P1 & $86.73(6)$ \\
Cl3-Tc1-P2 & $91.38(5)$ & C1-Tc1-Cl1 & $88.5(1)$ \\
P2-Tc1-P1 & $178.39(4)$ & C1-Tc1-Cl2 & $88.9(1)$ \\
\hline
\end{tabular}


Figure S4. Ellipsoid representation (50\% probability) of trans-[ $\left.\mathrm{TCNCl}_{2}\left(\mathrm{CNAr}^{\mathrm{Dipp} 2}\right)_{2}\right]$

Hydrogen atoms are omitted for clarity. Only one of two molecules contained in the asymmetric unit is depicted.

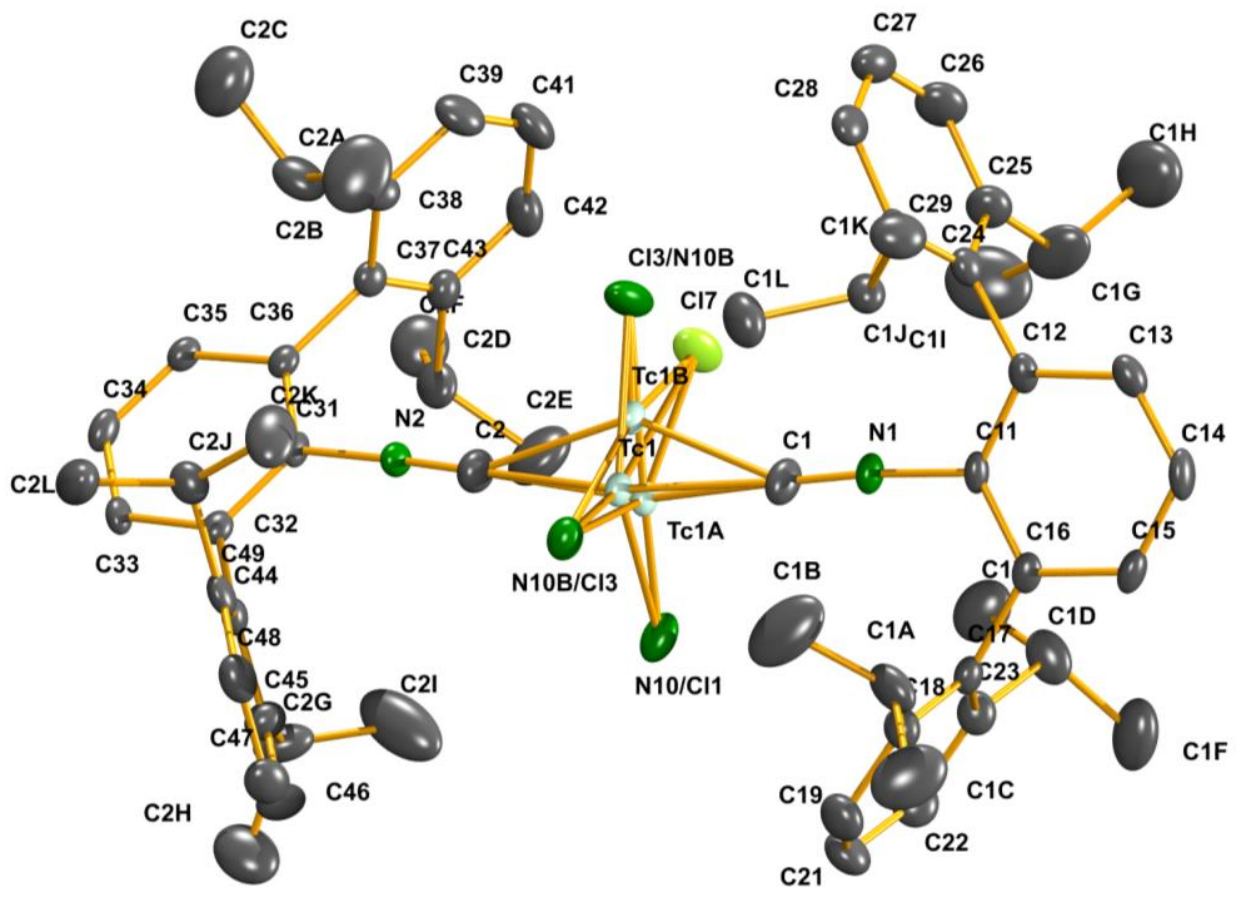

Table S5. Selected bond lengths $(\AA)$ and angles $\left(^{\circ}\right)$ in trans $-\left[\operatorname{TcNCl}_{2}\left(\mathrm{CNAr}^{\mathrm{Dipp} 2}\right)_{2}\right]$

\begin{tabular}{llll}
\hline Bond lengths & & & \\
\hline Tc1-C1 & $2.116(4)$ & Tc1-N10 & $1.683(2)$ \\
Tc1-C2 & $2.098(4)$ & Tc1B-N10B & $1.489(4)$ \\
C1-N1 & $1.192(2)$ & Tc1-Cl7 & $2.806(4)$ \\
C2-N2 & $1.147(4)$ & C11-N1 & $1.418(4)$ \\
Tc1A-N10A & $1.874(2)$ & C2-N2 & $1.147(4)$ \\
\hline Bond angles & & & \\
\hline N10-Tc1-C1 & $99.30(1)$ & N10B-Tc1B-Cl7 & $78.0(2)$ \\
Cl2-Tc1-N10 & $163.44(1)$ & C1-Tc1B-C2 & $141.3(2)$ \\
C1-Tc1-Cl7 & $80.25(14)$ & N1-C1-Tc1A & $165.8(4)$ \\
N10A-Tc1A-Cl7 & $115.98(15)$ & N1-C1-Tc1B & $153.5(4)$ \\
\hline
\end{tabular}


Figure S5. Ellipsoid representation (50\% probability) of trans-[Tc(NPh) $\left.\mathrm{Cl}_{3}\left(\mathrm{CNAr}^{\mathrm{Dipp} 2}\right)_{2}\right]$-toluene Hydrogen atoms are omitted for clarity.

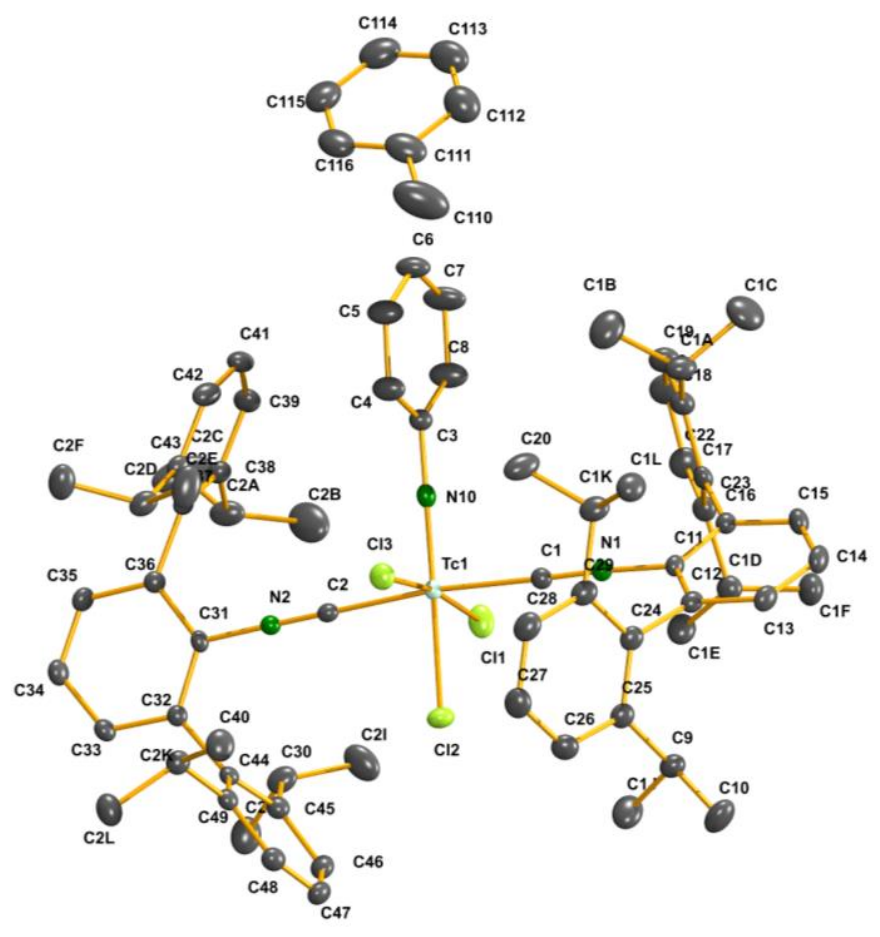

Table S6. Selected bond lengths $(\AA)$ and angles $\left(^{\circ}\right)$ in trans-[Tc(NPh $\left.\mathrm{Cl}_{3}\left(\mathrm{CNArDipp2}_{2}\right)_{2}\right]$ toluene

\begin{tabular}{llll}
\hline Bond lengths, distances & & \\
\hline Tc1-N10 & $1.705(6)$ & Tc1-Cl1 & $2.391(6)$ \\
Tc1-C1 & $2.090(1)$ & Tc1-Cl2 & $2.383(6)$ \\
Tc1-C2 & $2.081(1)$ & Tc1-Cl3 & $2.432(5)$ \\
C1-N1 & $1.151(2)$ & Tc1-N10 & $1.710(1)$ \\
\hline Bond angles & & & \\
\hline Cl2-Tc1-Cl1 & $88.55(2)$ & N10-Tc1-Cl1 & $98.54(4)$ \\
Cl3-Tc1-Cl1 & $171.95(1)$ & N10-Tc1-Cl2 & $172.72(4)$ \\
Cl2-Tc1-Cl3 & $83.59(2)$ & N10-Tc1-C3 & $89.37(4)$ \\
N10-Tc1-C1 & $90.89(5)$ & C1-Tc1-Cl1 & $89.89(4)$ \\
N10-Tc1-C2 & $94.80(5)$ & C2-Tc1-Cl1 & $87.63(4)$ \\
N1-C1-Tc1 & $177.50(1)$ & C1-Tc1-Cl2 & $87.47(4)$ \\
N2-C2-Tc1 & $177.95(1)$ & C1-Tc1-Cl3 & $91.47(4)$ \\
\hline
\end{tabular}


Figure S6. Ellipsoid representation (50\% probability) of trans-[Tc(NPh) $\left.\mathrm{Br}_{3}\left(\mathrm{CNAr}^{\mathrm{Dipp} 2}\right)_{2}\right]$-toluene Hydrogen atoms are omitted for clarity.

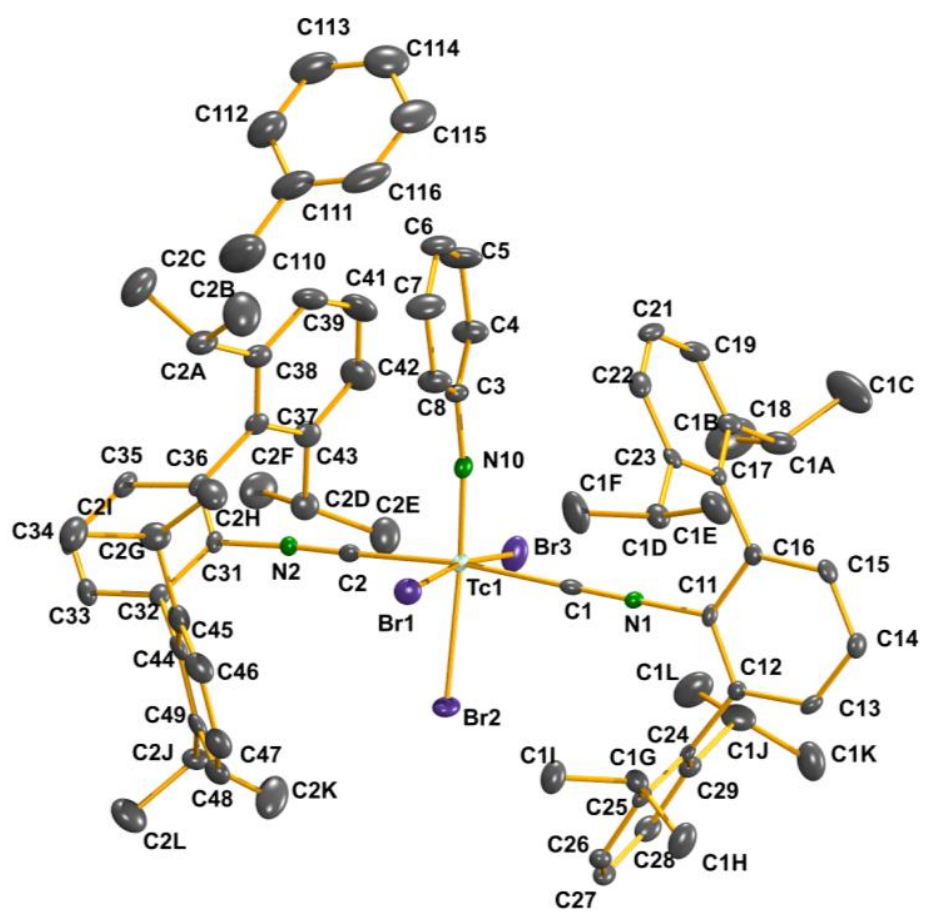

Table S7. Selected bond lengths $(\AA \AA)$ and angles $\left(^{\circ}\right)$ in trans- $\left[\mathrm{Tc}(\mathrm{NPh}) \mathrm{Br}_{3}\left(\mathrm{CNAr}^{\mathrm{Dipp} 2}\right)_{2}\right] \cdot$ toluene

\begin{tabular}{llll}
\hline Bond lengths & & & \\
\hline Tc1-N10 & $1.708(2)$ & Tc1-Br1 & $2.5657(4)$ \\
Tc1-C1 & $2.074(3)$ & Tc1-Br2 & $2.5333(4)$ \\
Tc1-C2 & $2.079(3)$ & Tc1-Br3 & $2.5299(4)$ \\
C1-N1 & $1.150(3)$ & Tc1-N10 & $1.708(2)$ \\
\hline Bond angles & & & \\
\hline Br2-Tc1-Br1 & $83.539(1)$ & N10-Tc1-Br1 & $89.54(7)$ \\
Br3-Tc1-Br1 & $172.24(1)$ & N10-Tc1-Br2 & $172.95(7)$ \\
Br2-Tc1-Br3 & $88.88(1)$ & N10-Tc1- & $98.07(7)$ \\
N10-Tc1-C1 & $95.6(1)$ & C1-Tc1-Br1 & $90.45(7)$ \\
N10-Tc1-C2 & $92.0(1)$ & C2-Tc1-Br1 & $91.49(7)$ \\
N1-C1-Tc1 & $177.3(2)$ & C1-Tc1-Br2 & $85.82(7)$ \\
N2-C2-Tc1 & $177.7(2)$ & C1-Tc1-Br3 & $87.38(7)$ \\
\hline
\end{tabular}


Figure S7. Ellipsoid representation (50\% probability) of mer-[Tc(PPhMe$\left.)_{2} \mathrm{Cl}_{3}\left(\mathrm{CNAr}^{\mathrm{Mes} 2}\right)\right]$. Hydrogen atoms are omitted for clarity.

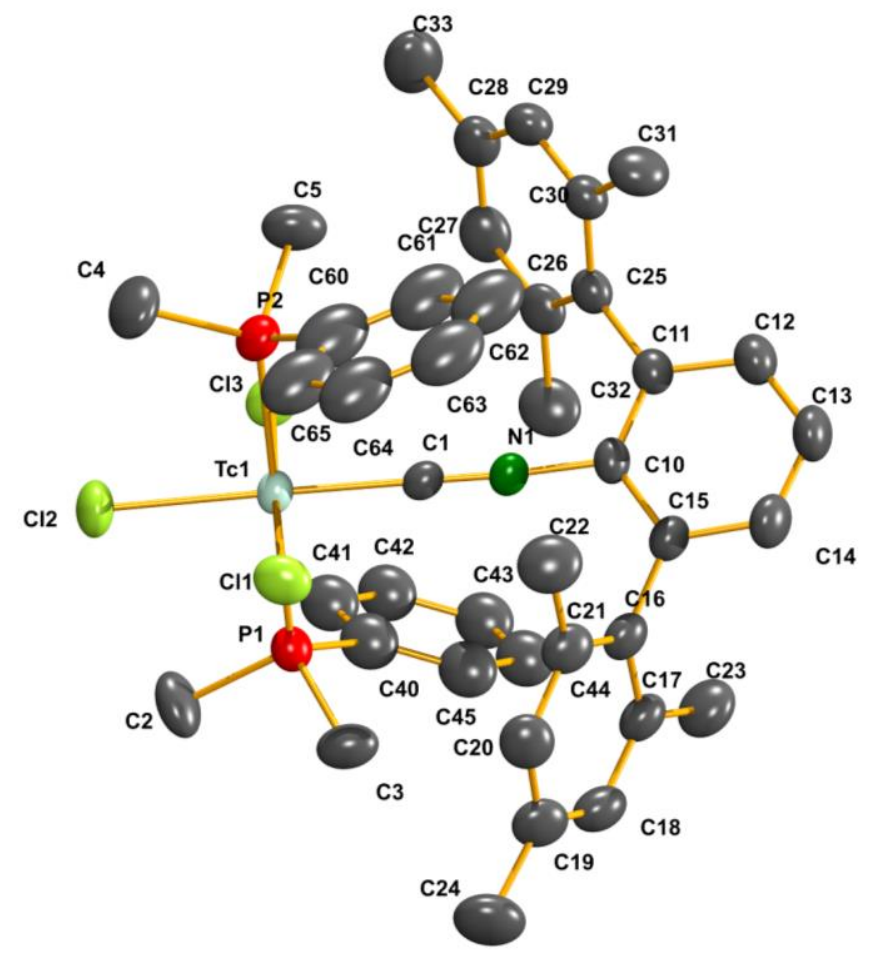

Table S8. Selected bond lengths $(\AA)$ and bond angles $\left(^{\circ}\right)$ in mer-[Tc(PPhMe 2$\left.)_{2} \mathrm{Cl}_{3}\left(\mathrm{CNAr}^{\mathrm{Mes} 2}\right)\right]$.

\begin{tabular}{llll}
\hline Bond lengths & & & \\
\hline Tc1-C1 & $2.003(3)$ & Tc1-Cl1 & $2.330(1)$ \\
Tc1-P1 & $2.467(1)$ & Tc1-Cl2 & $2.439(1)$ \\
Tc1-P2 & $2.469(1)$ & Tc1-Cl3 & $2.338(13)$ \\
C1-N1 & $2.469(1)$ & N1-C10 & $1.403(4)$ \\
\hline Bond angles & & & \\
\hline Cl1-Tc1-Cl3 & $90.35(4)$ & Cl2-Tc1-Cl1 & $91.39(5)$ \\
Cl1-Tc1-P1 & $89.89(4)$ & Cl2-Tc1-Cl3 & $90.35(4)$ \\
Cl2-Tc1-P2 & $90.12(4)$ & Cl2-Tc1-P1 & $89.71(4)$ \\
N1-C1-Tc1 & $179.6(4)$ & Cl3-Tc1-P1 & $91.17(4)$ \\
Cl3-Tc1-P2 & $90.43(4)$ & C1-Tc1-Cl1 & $88.06(10)$ \\
P2-Tc1-P1 & $178.39(4)$ & C1-Tc1-Cl2 & $179.20(10)$ \\
\hline
\end{tabular}


Figure S8. Ellipsoid representation (50\% probability) of cis-[ $\left.\mathrm{TcNCl}_{2}\left(\mathrm{CNAr}^{\mathrm{Mes} 2}\right)_{2}(\mathrm{MeOH})\right]$. Hydrogen atoms are omitted for clarity.

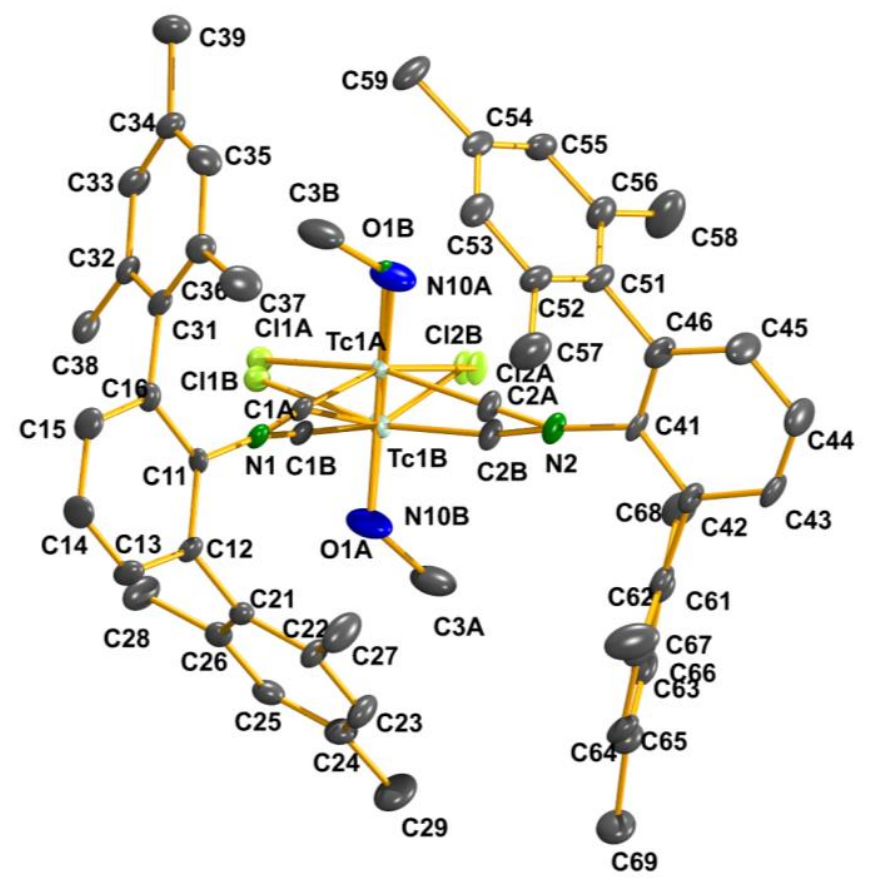

Table S9. Selected bond lengths, distances $(\AA)$, and bond angles $\left(^{\circ}\right)$ in $c i s-\left[\operatorname{TcNCl}_{2}\left(\mathrm{CNArMes}^{2}\right)_{2}-\right.$ $(\mathrm{MeOH})]$.

\begin{tabular}{llll}
\hline Bond lengths & & \\
\hline Tc1A-Cl1A & $2.413(1)$ & Tc1A-C2A & $2.039(1)$ \\
Tc1A-Cl2 & $2.390(1)$ & C1A-N1 & $1.19(2)$ \\
Tc1A-N10A & $1.621(1$ & C2A-N2 & $1.16(1)$ \\
Tc1A-Cl1A & $2.413(1)$ & Tc1A-C2A & $2.039(1)$ \\
\hline Bond angles & & & \\
\hline N2-C2A-Tc1A & $175.5(9)$ & Cl2A-Tc1A-Cl1A & $89.6(3)$ \\
C1A-Tc1A-O11A & $77.9(4)$ & N10A-Tc1A-Cl1A & $102.9(4)$ \\
C2A-Tc1A-O1A & $81.9(5)$ & N10A-Tc1A-Cl2A & $104.8(5)$ \\
C1A-Tc1A-C2A & $92.5(4)$ & N10A-Tc1A-C1A & $92.4(5)$ \\
N10A-Tc1A-O1A & $168.6(6)$ & N10A-Tc1A-C2A & $92.8(5)$ \\
Cl1A-Tc1A-O1A & $83.4(4)$ & N1-C1A-Tc1A & $176.1(7)$ \\
\hline
\end{tabular}


Figure S9. Ellipsoid representation (50\% probability) of cis-[ $\left[\mathrm{TCNCl}_{2}\left(\mathrm{CNArMes}_{2}\right)_{2}\left(\mathrm{H}_{2} \mathrm{O}\right)\right]$. Hydrogen atoms are omitted for clarity.

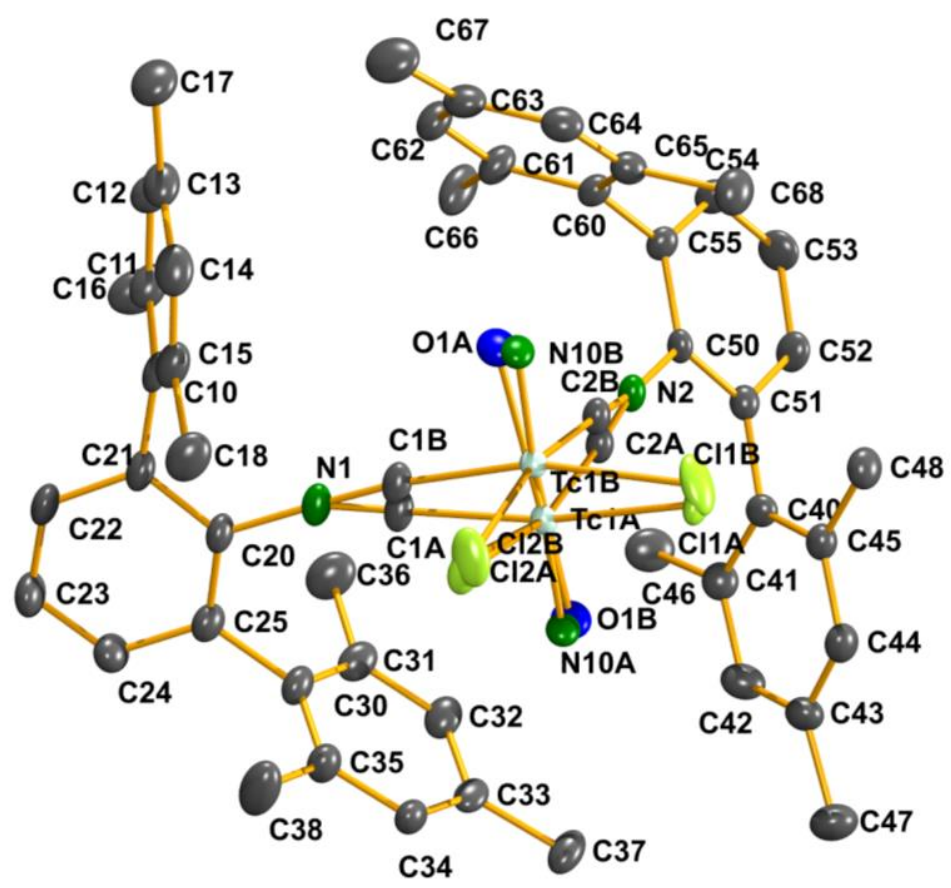

Table S12. Selected bond lengths $(\AA)$ and angles $\left(^{\circ}\right)$ in cis- $\left[\operatorname{TcNCl}_{2}\left(\mathrm{CNArMes}_{2}\right)_{2}\left(\mathrm{H}_{2} \mathrm{O}\right)\right]$.

\begin{tabular}{llll}
\hline Bond lengths & & \\
\hline Tc1A-Cl1A & $2.35(2)$ & Tc1A-C2A & $2.062(1)$ \\
Tc1A-Cl2A & $2.343(2)$ & C1A-N1 & $1.133(2)$ \\
Tc1A-N10A & $1.620(2)$ & C2A-N2 & $1.15(1)$ \\
Tc1A-Cl1A & $2.068(2)$ & Tc1A-C2A & $2.51(2)$ \\
\hline Bond angles & & & \\
\hline N2-C2A-Tc1A & $172.5(1)$ & Cl2A-Tc1-Cl1A & $91.4(7)$ \\
C1A-Tc1A-O1A & $78.2(5)$ & N10A-Tc1A-Cl1A & $97.5(1)$ \\
C2A-Tc1A-O1A & $81.5(7)$ & N10A-Tc1A-Cl2A & $97.3(7)$ \\
C1A-Tc1A-C2A & $92.5(6)$ & N10A-Tc1A-C1A & $94.1(1)$ \\
N10A-Tc1-O1A & $171.2(8)$ & N10A-Tc1A-C2A & $94.6(6)$ \\
Cl1A-Tc1A-O1A & $90.5(5)$ & N1-C1A-Tc1A & $173.2(1)$ \\
\hline
\end{tabular}




\section{Spectroscopic Data}

Figure S10. ${ }^{1} \mathrm{H}$ NMR spectrum of trans- $\left[\mathrm{Tc}(\mathrm{CO}){ }_{3} \mathrm{Cl}\left(\mathrm{CNAr}^{\mathrm{Dipp} 2}\right)_{2}\right]$

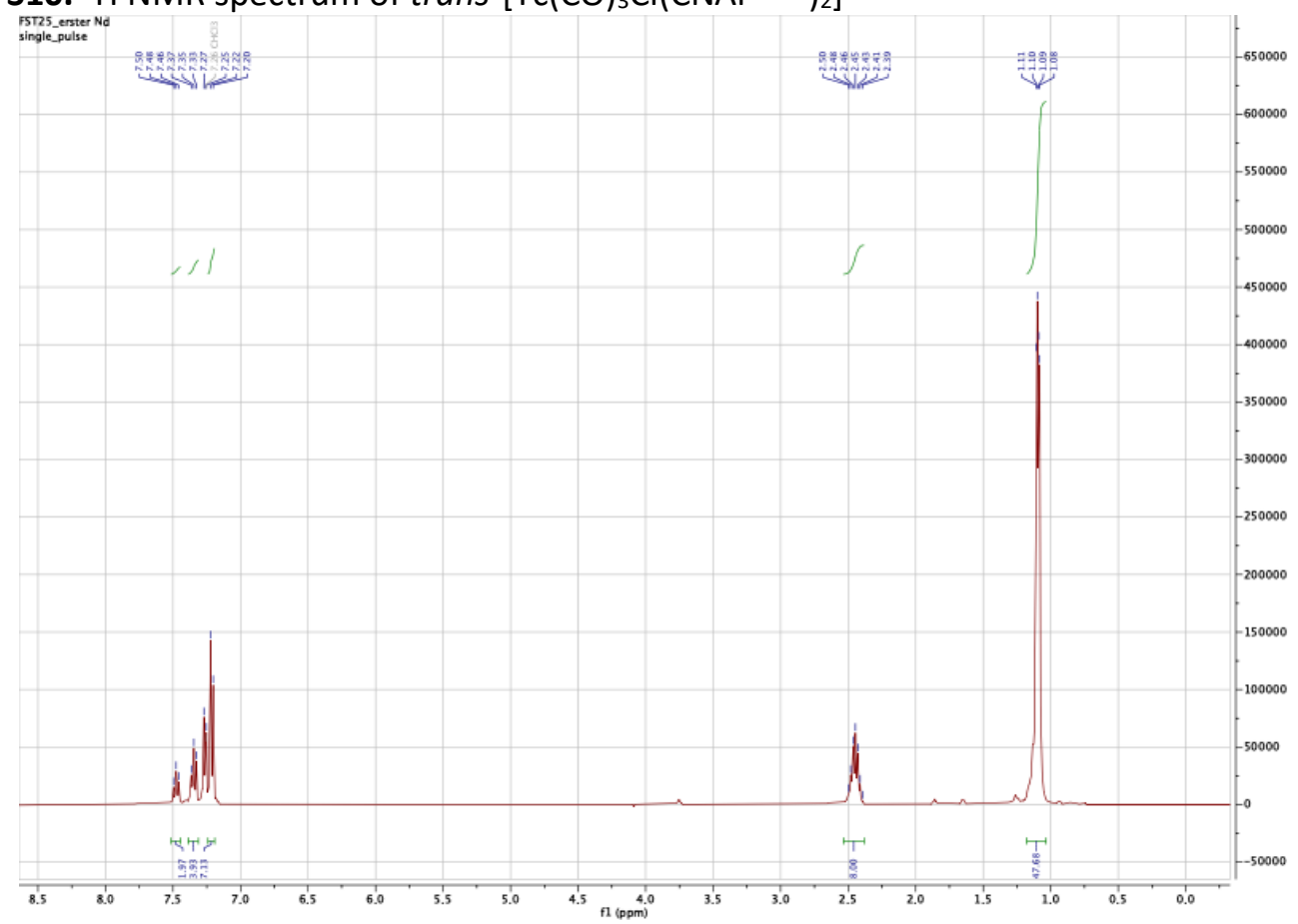

Figure S11. ${ }^{99} \mathrm{Tc} N \mathrm{NMR}$ spectrum of trans- $\left[\mathrm{Tc}(\mathrm{CO})_{3} \mathrm{Cl}\left(\mathrm{CNAr}{ }^{\mathrm{Dipp} 2}\right)_{2}\right]$

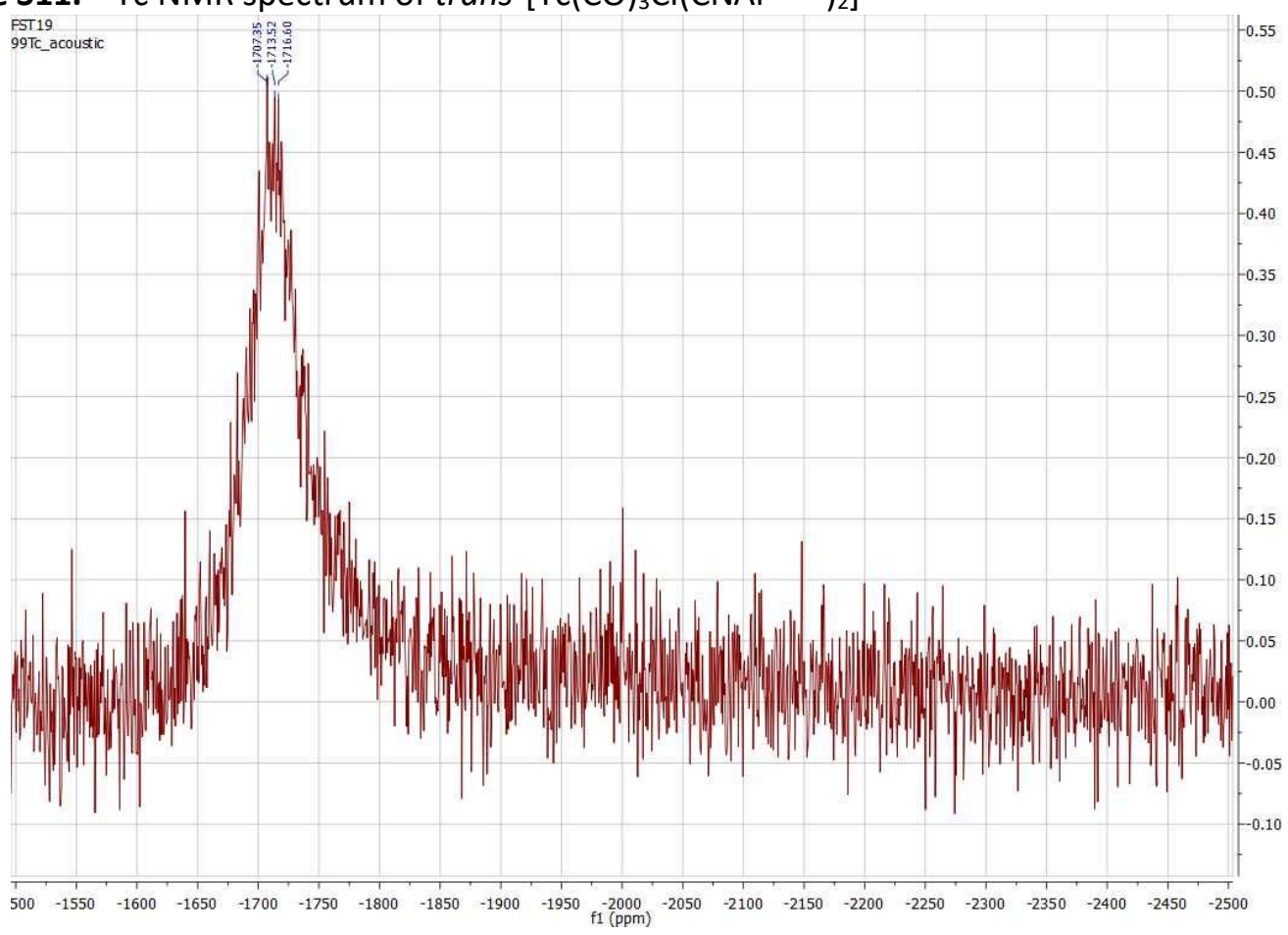


Figure S12. IR spectrum of a raw sample of trans, mer- $\left[\mathrm{Tc}(\mathrm{CO})_{3} \mathrm{Cl}\left(\mathrm{CNAr}{ }^{\mathrm{Dipp} 2}\right)_{2}\right]$. The band at $2181 \mathrm{~cm}-$ 1 does not belong to the complex or a possible cis, fac- $\left[\mathrm{Tc}(\mathrm{CO})_{3} \mathrm{Cl}\left(\mathrm{CNAr}{ }^{\mathrm{Dipp} 2}\right)_{2}\right]$ isomer as can be concluded from the data obtained for the corresponding Re compounds described in ref. 39.

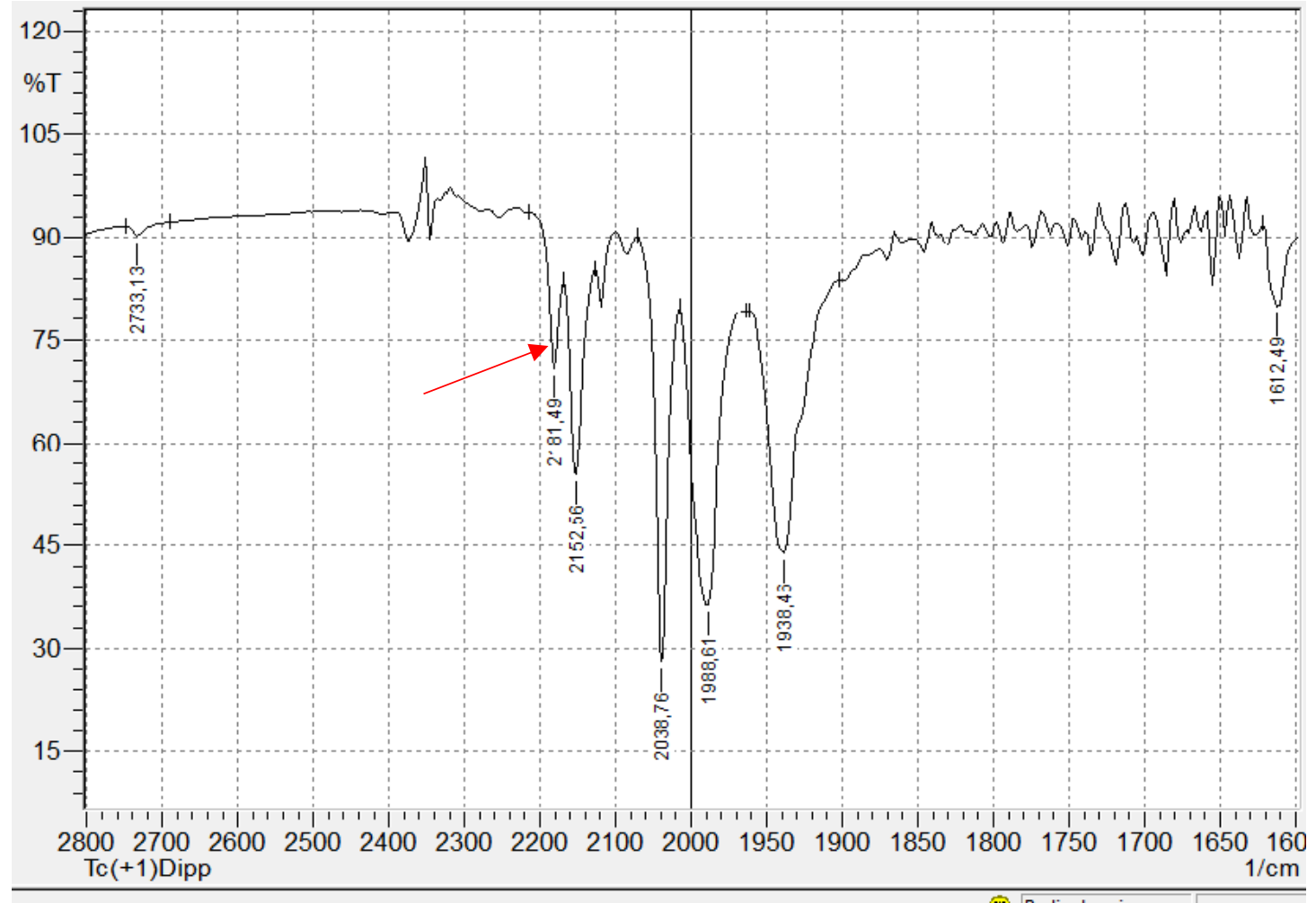

Figure S13. ${ }^{1} \mathrm{H}$ NMR spectrum of trans-[Tc(NO) $\left.\mathrm{Cl}_{2}\left(\mathrm{PPh}_{3}\right)(\mathrm{CNArDipp2})_{2}\right]$.

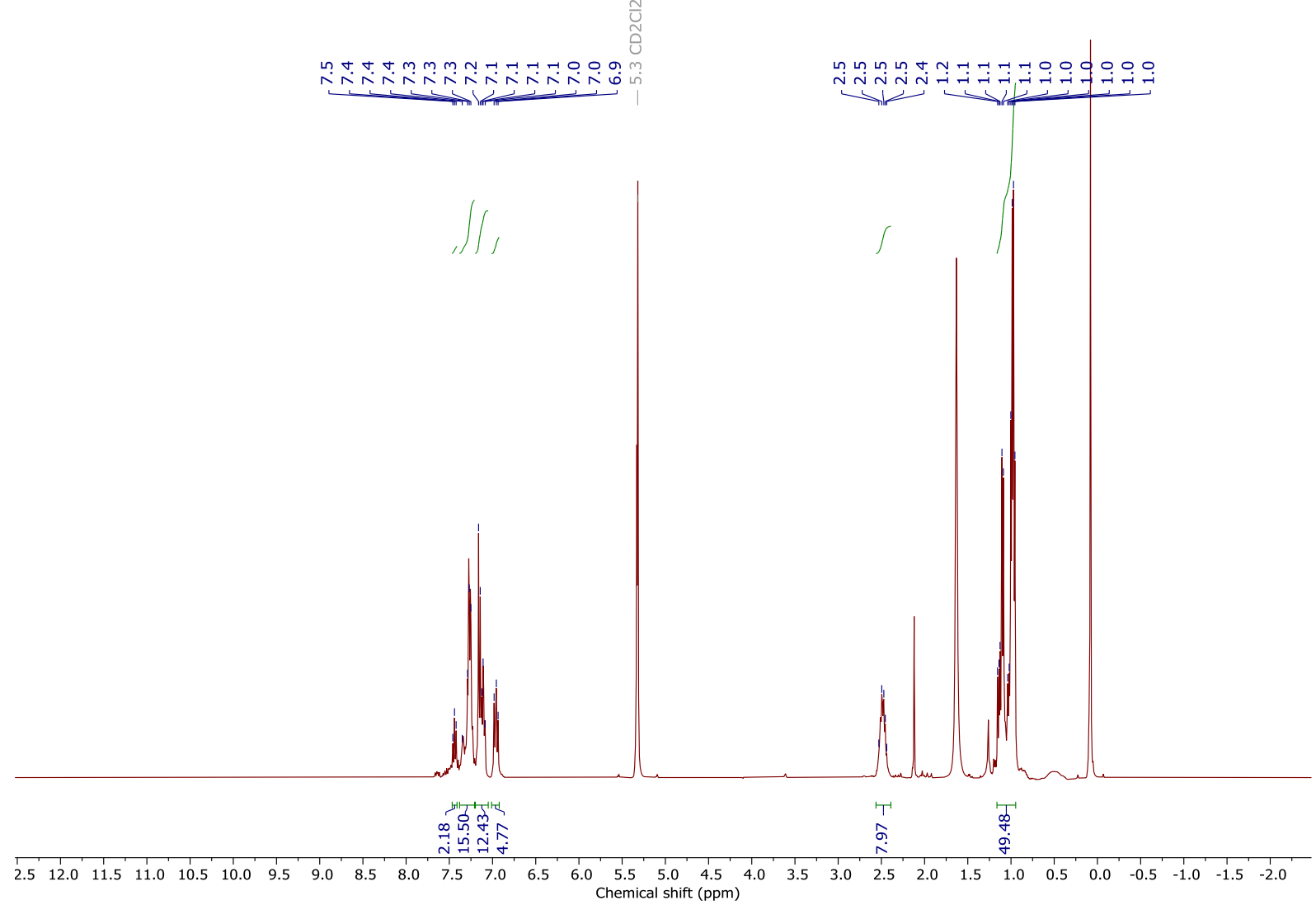


Figure S14. ${ }^{99} \mathrm{Tc} \mathrm{NMR}$ spectrum of $\left[\mathrm{Tc}(\mathrm{NO}) \mathrm{Cl}_{2}\left(\mathrm{PPh}_{3}\right)\left(\mathrm{CNAr}{ }^{\mathrm{Dipp} 2}\right)_{2}\right]$. $\stackrel{\text { in }}{i}$

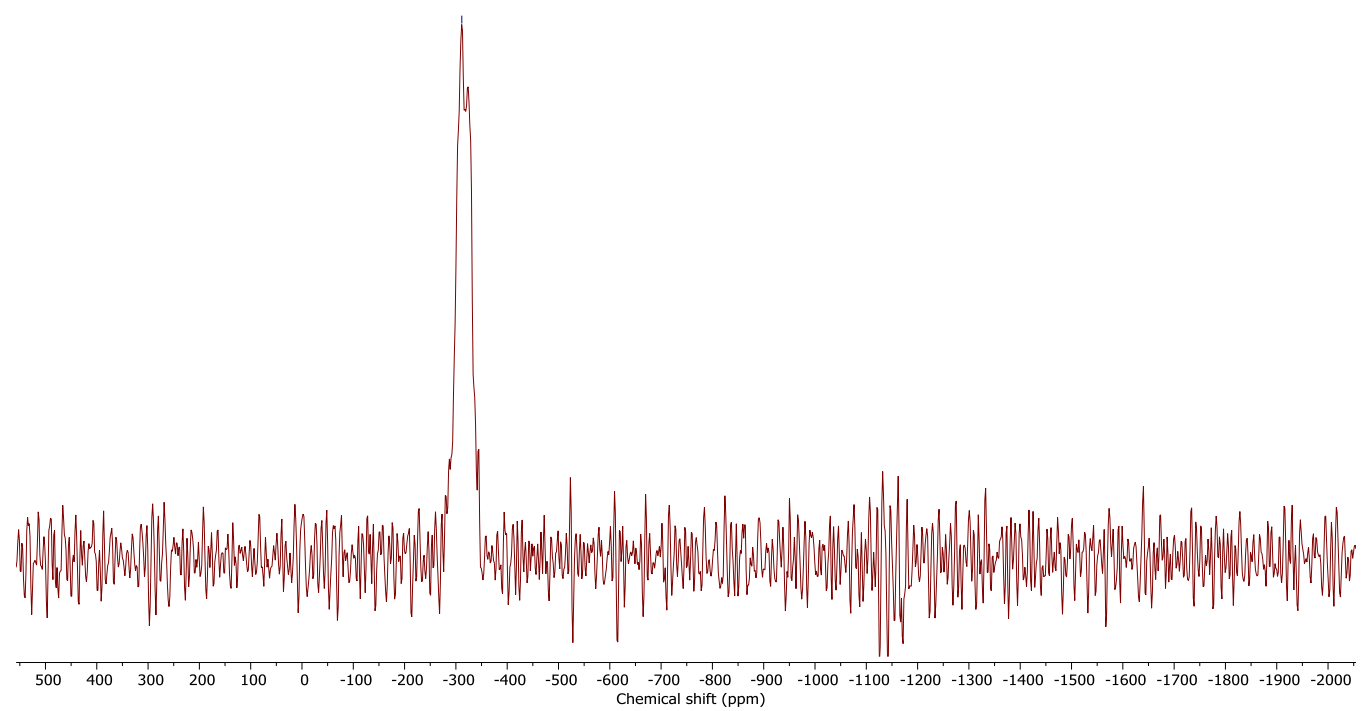

Figure S15. IR spectrum of $\left[\mathrm{Tc}(\mathrm{NO}) \mathrm{Cl}_{2}\left(\mathrm{PPh}_{3}\right)\left(\mathrm{CNArDipp2}_{2}\right)_{2}\right]$.

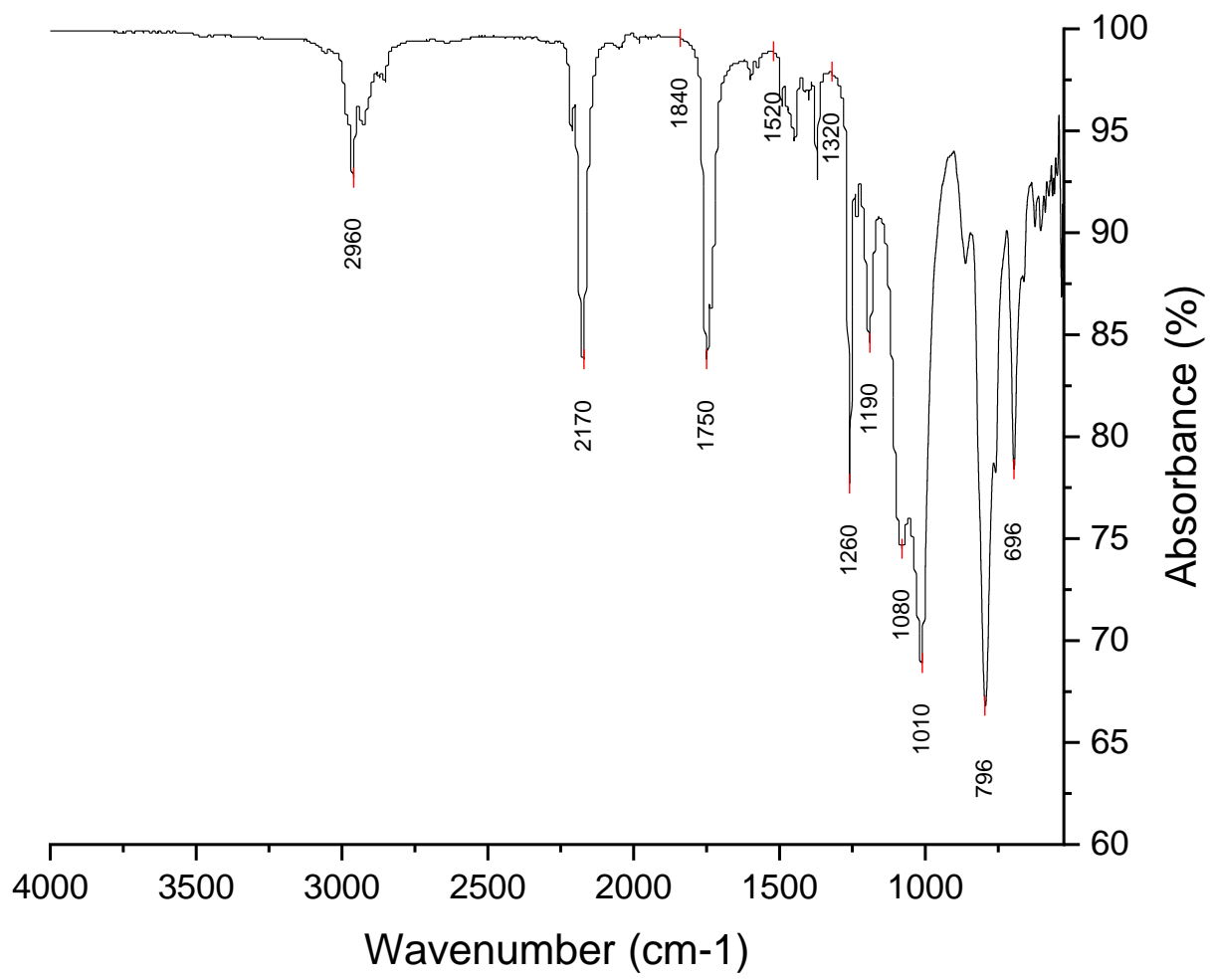


Figure S16. IR spectrum of $m e r-\left[\mathrm{TcCl}_{3}\left(\mathrm{PPhMe}_{2}\right)_{2}\left(\mathrm{CNArDipp}_{2}\right)_{2}\right]$.

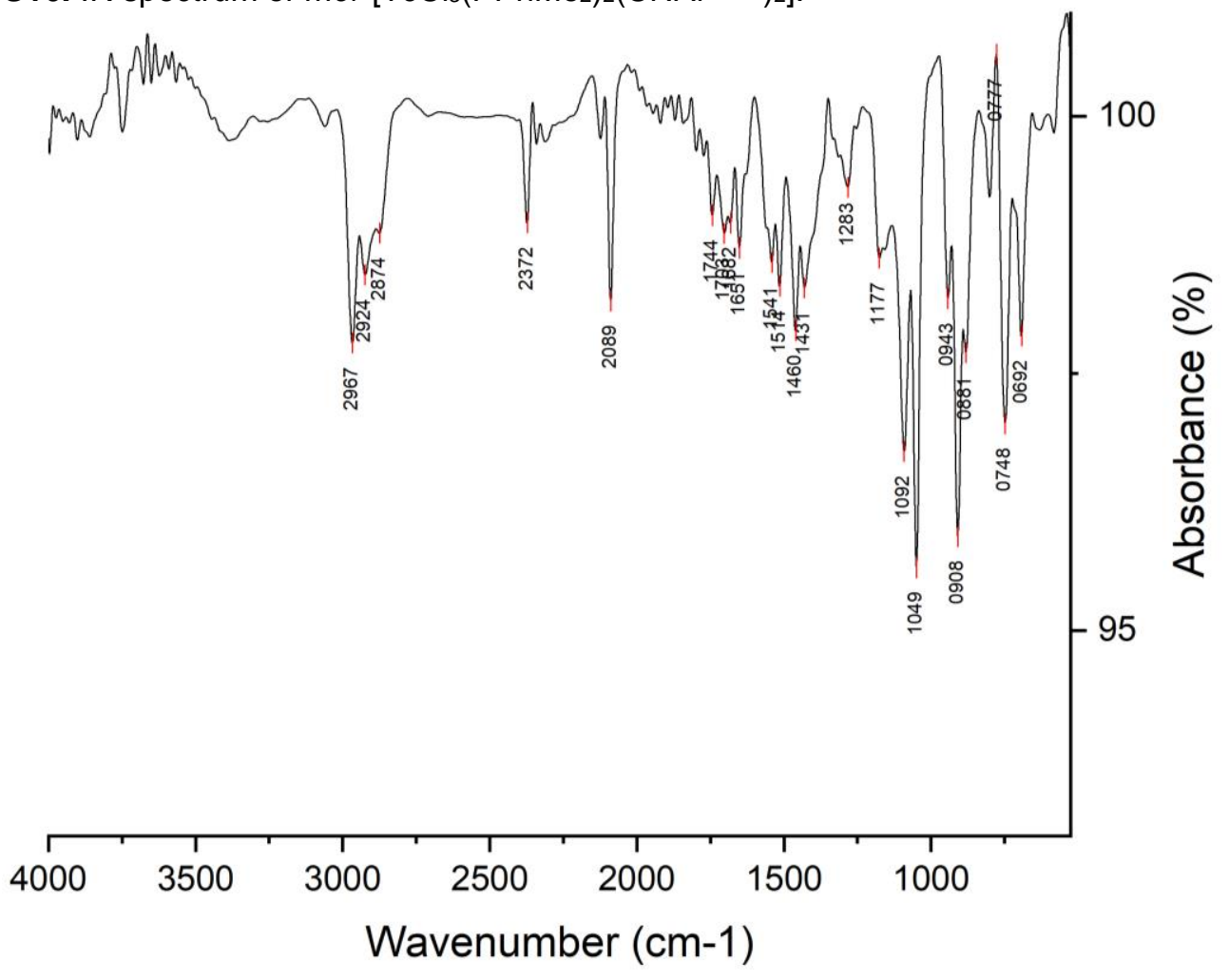

Figure S17. ${ }^{1} \mathrm{H}$ NMR spectrum of trans-[ $\left.\mathrm{TcNCl}_{2}\left(\mathrm{CNArDipp}_{2}\right)_{2}\right]$.

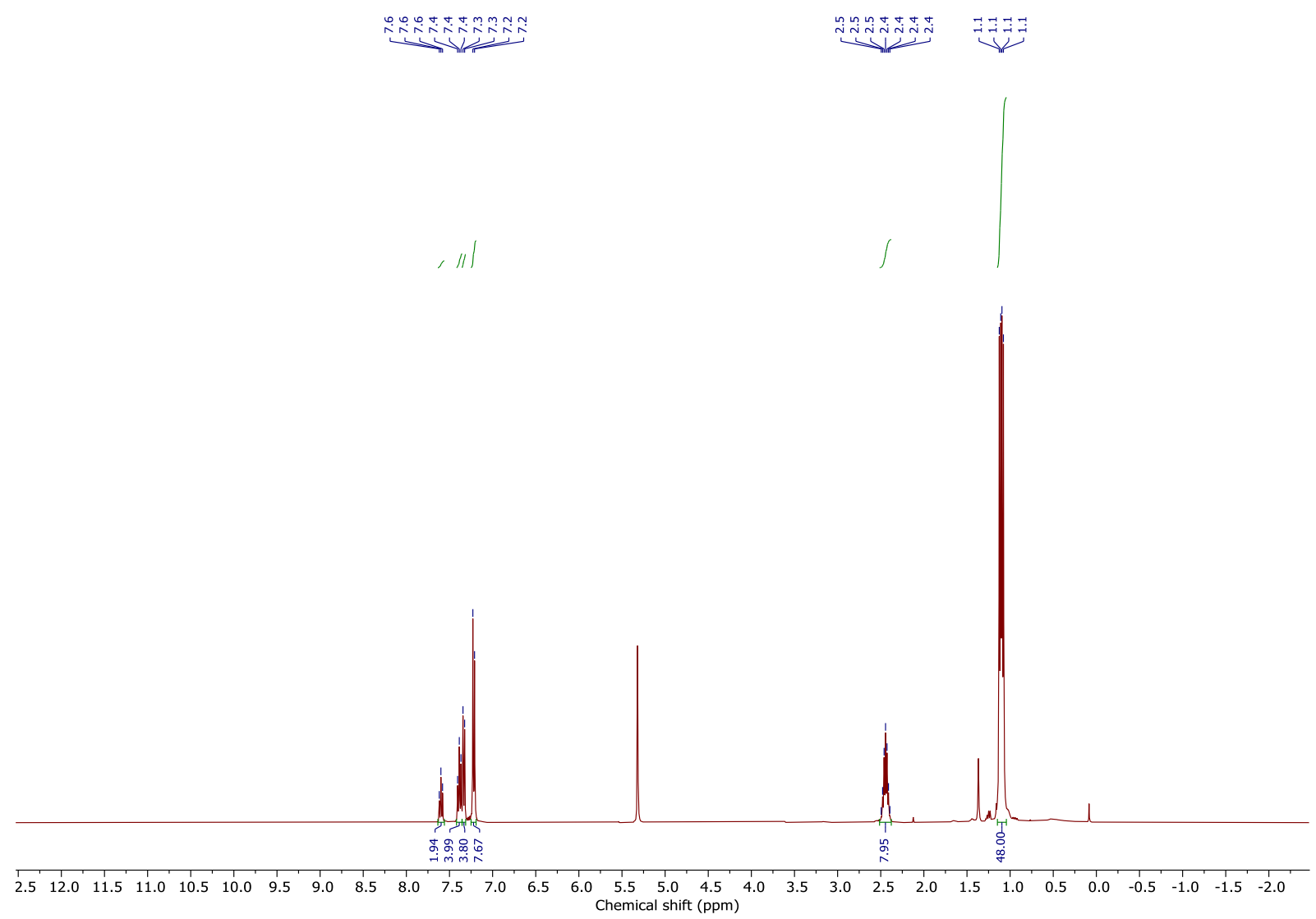


Figure S18. IR spectrum of trans-[ $\left.\mathrm{TcNCl}_{2}\left(\mathrm{CNAr}^{\mathrm{Dipp} 2}\right)_{2}\right]$.

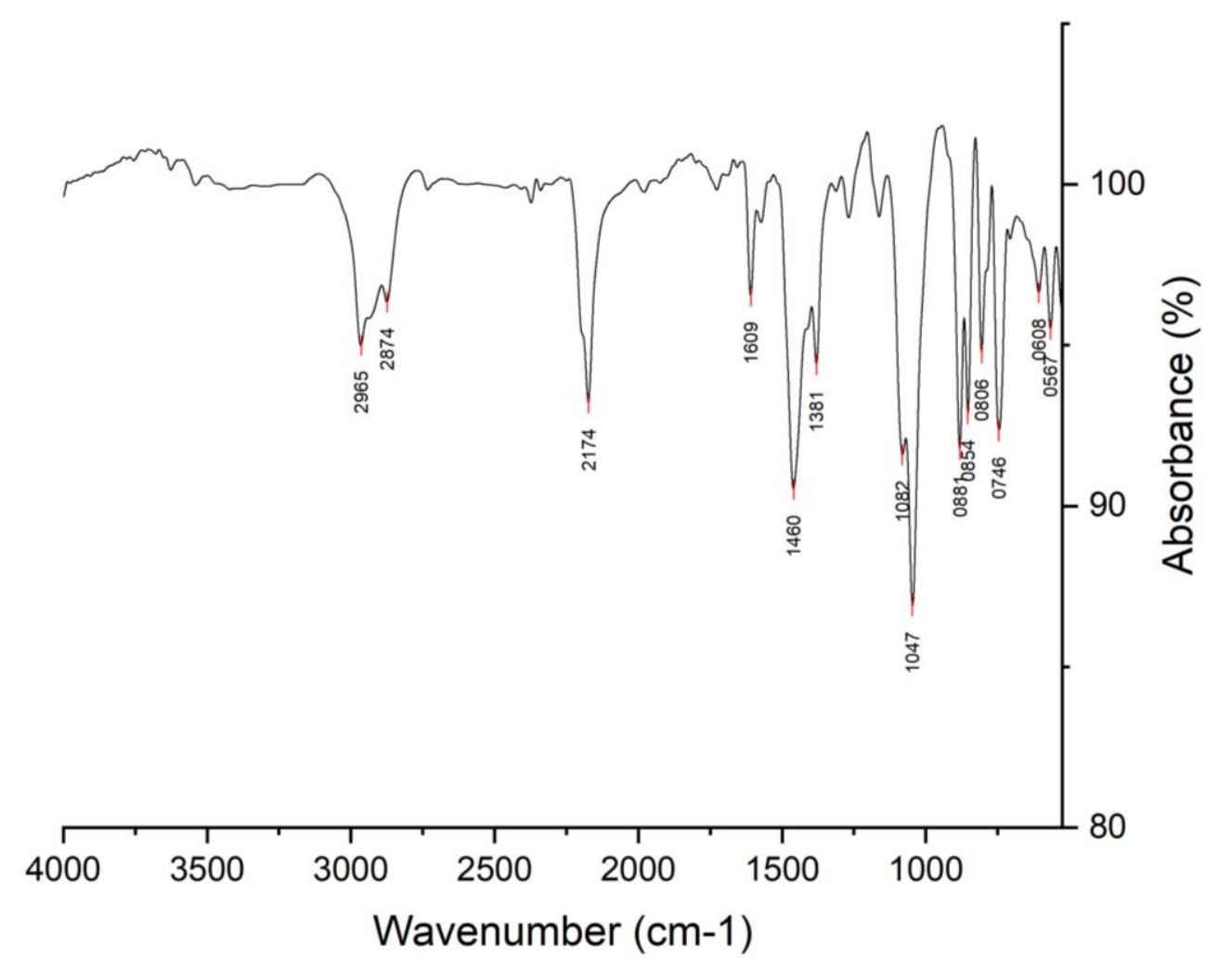

Figure S19. ${ }^{1} \mathrm{H}$ NMR spectrum of trans-[ $\left.\operatorname{TcNBr}_{2}\left(\mathrm{CNAr}^{\mathrm{Dipp} 2}\right)_{2}\right]$.

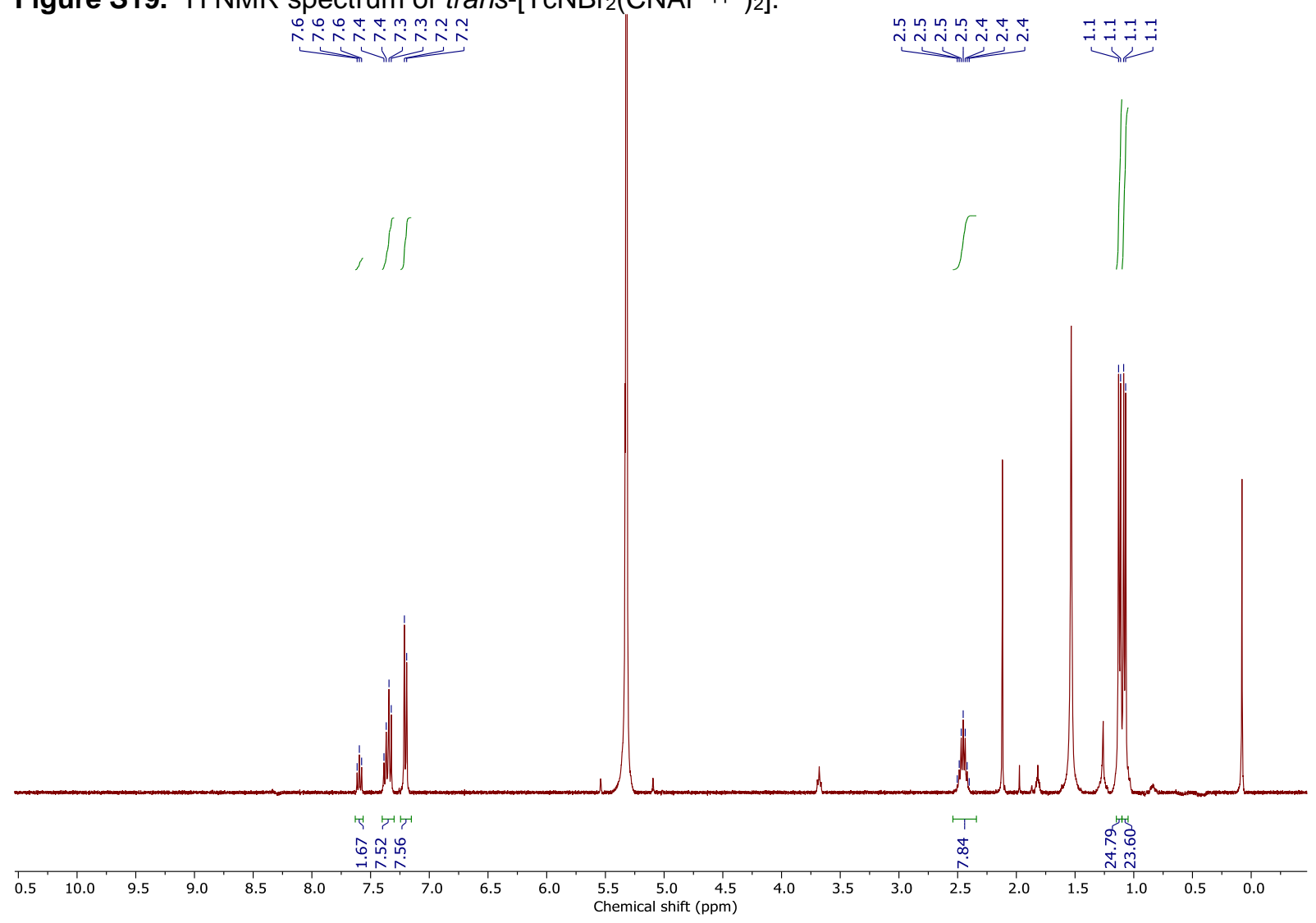


Figure S20. IR spectrum of trans-[TcNBr2 $\left.\left(\mathrm{CNAr}^{\mathrm{Dipp} 2}\right)_{2}\right]$.

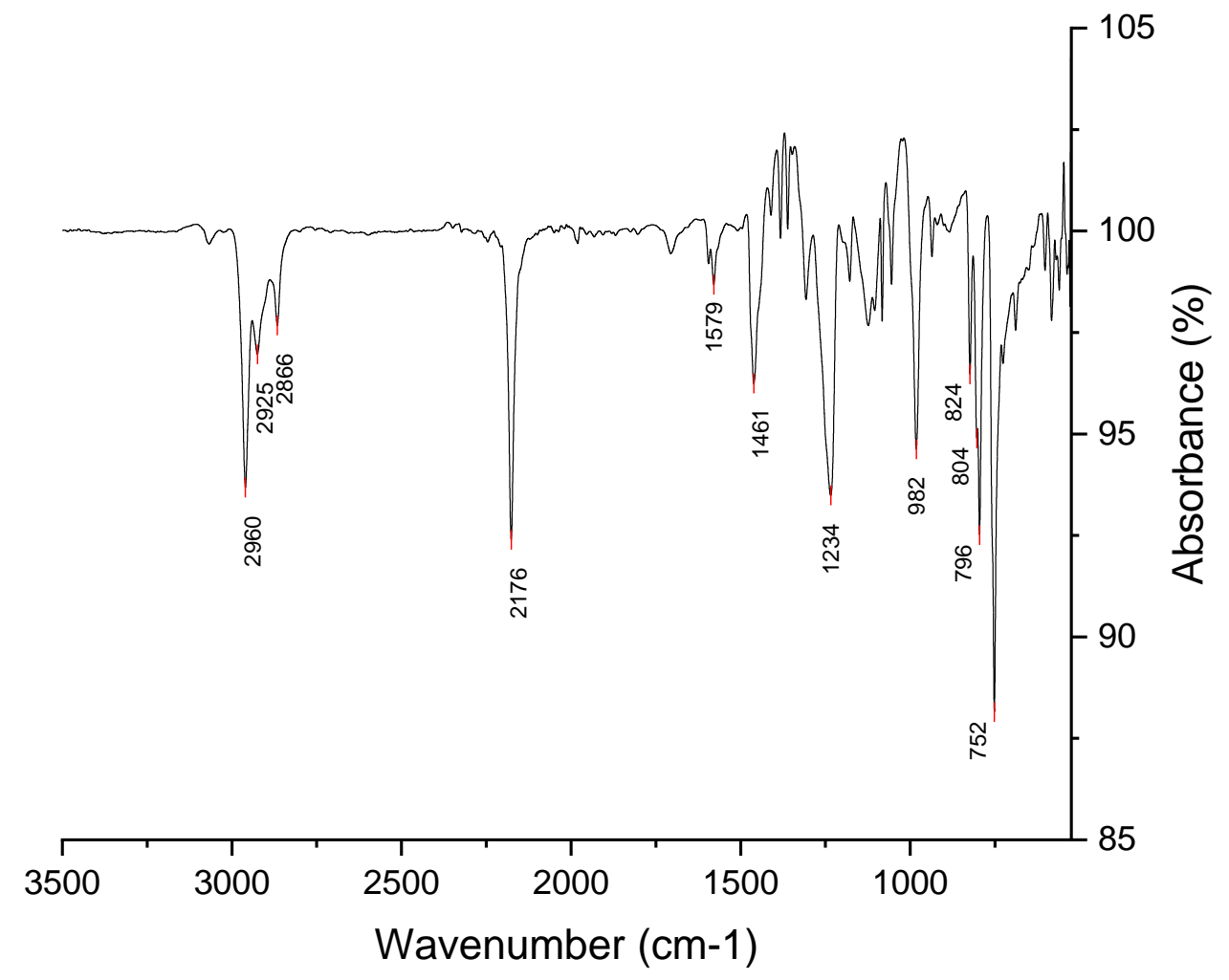

Figure S21. ${ }^{1} \mathrm{H}$ NMR spectrum of $m e r-\left[\mathrm{Tc}(\mathrm{NPh}) \mathrm{Cl}_{3}\left(\mathrm{CNArDipp}^{2}\right)_{2}\right]$.

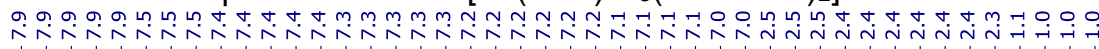

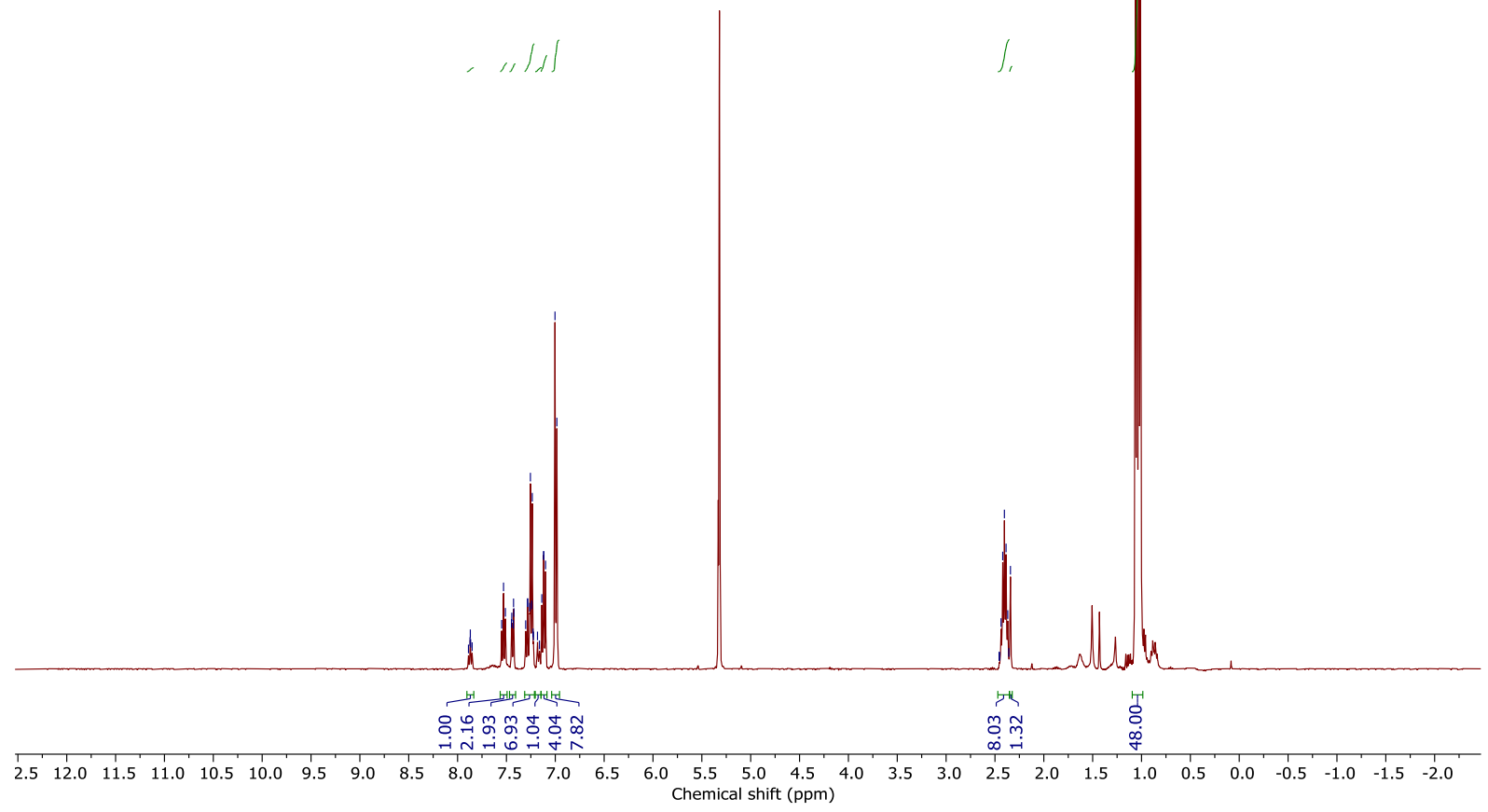


Figure S22. IR spectrum of $\operatorname{mer}\left[\mathrm{TC}(\mathrm{NPh}) \mathrm{Cl}_{3}\left(\mathrm{CNArDipp}_{2}\right)_{2}\right]$.

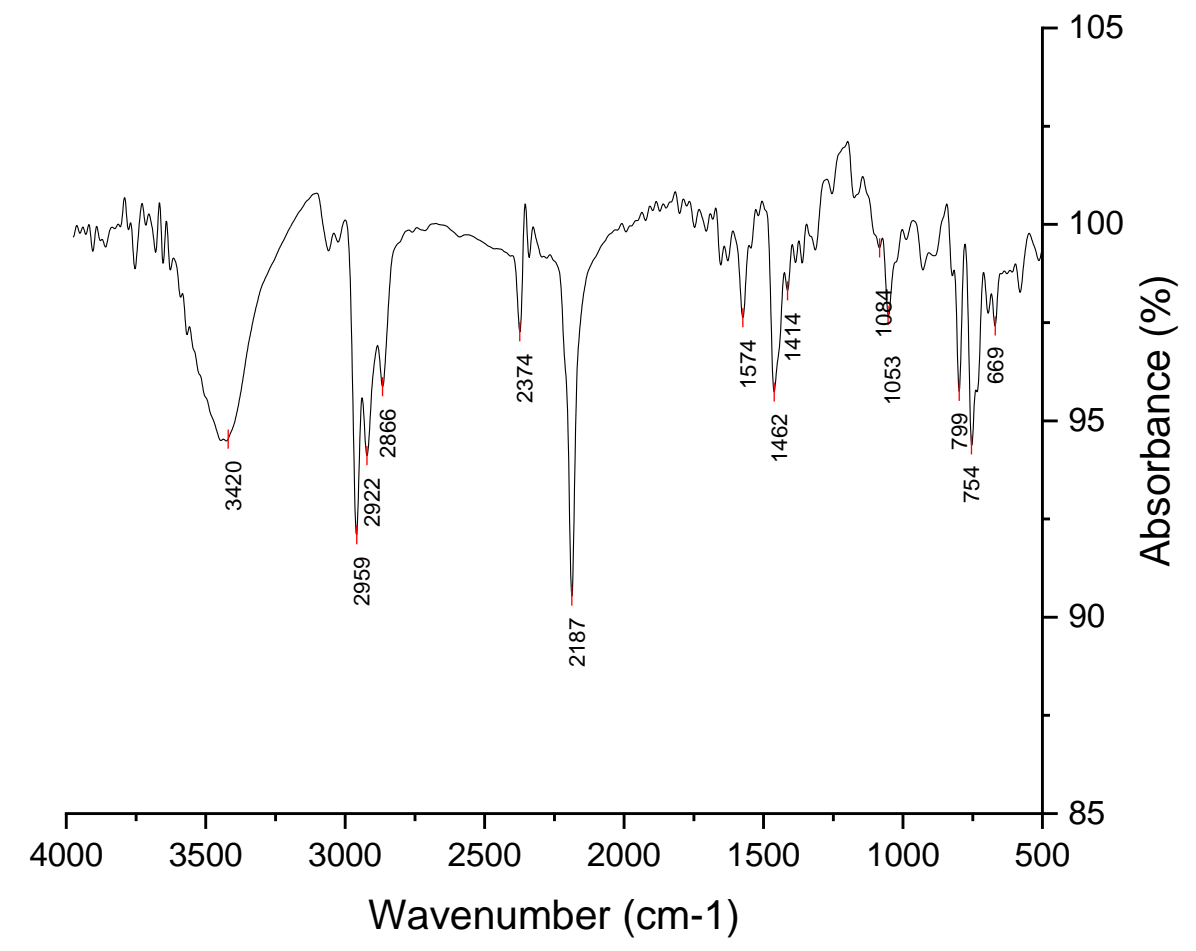

Figure S23. IR spectrum of $m e r-\left[\mathrm{Tc}(\mathrm{NPh}) \mathrm{Br}_{3}\left(\mathrm{CNAr}^{\mathrm{Dipp} 2}\right)_{2}\right]$.

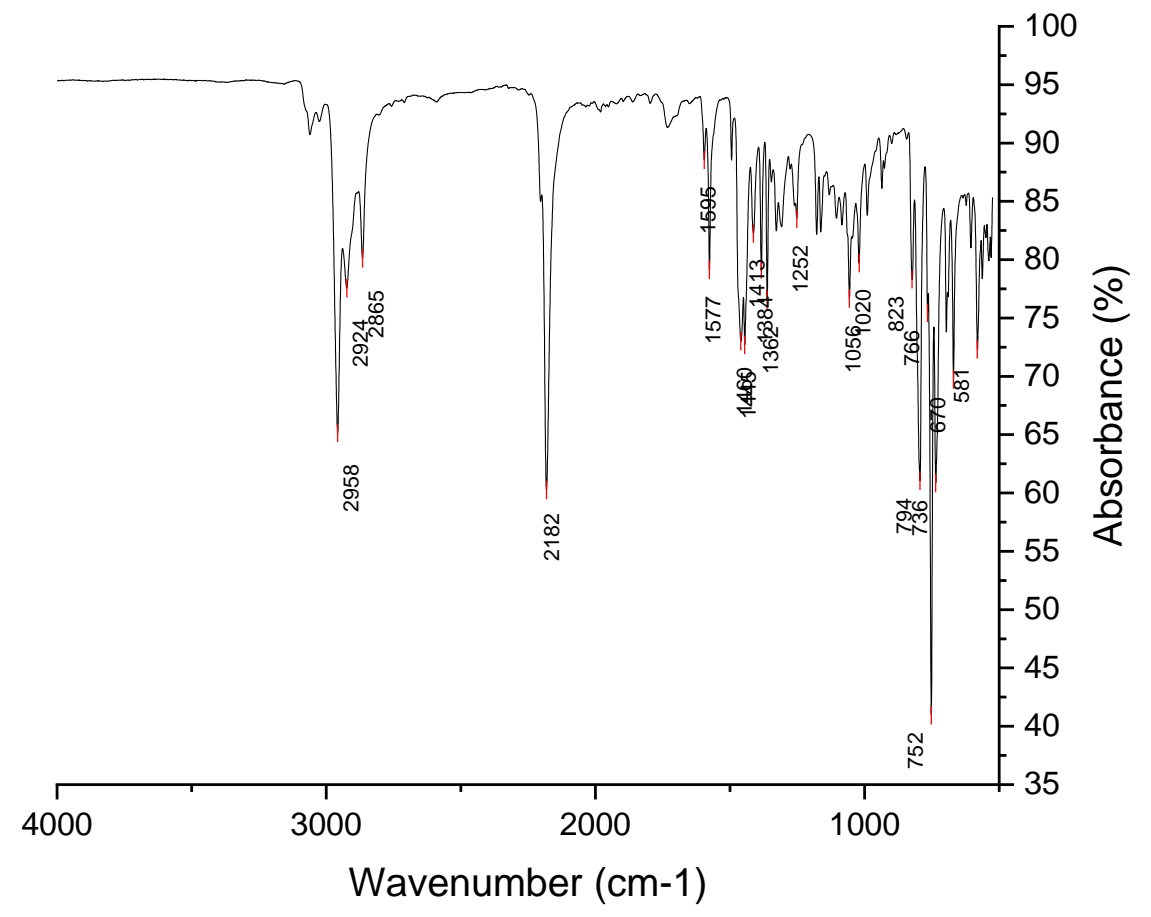


Figure S24. IR spectrum of $m e r-\left[\mathrm{TcCl}_{3}\left(\mathrm{PPhMe}_{2}\right)_{2}\left(\mathrm{CNArMes}^{2}\right)_{2}\right]$.

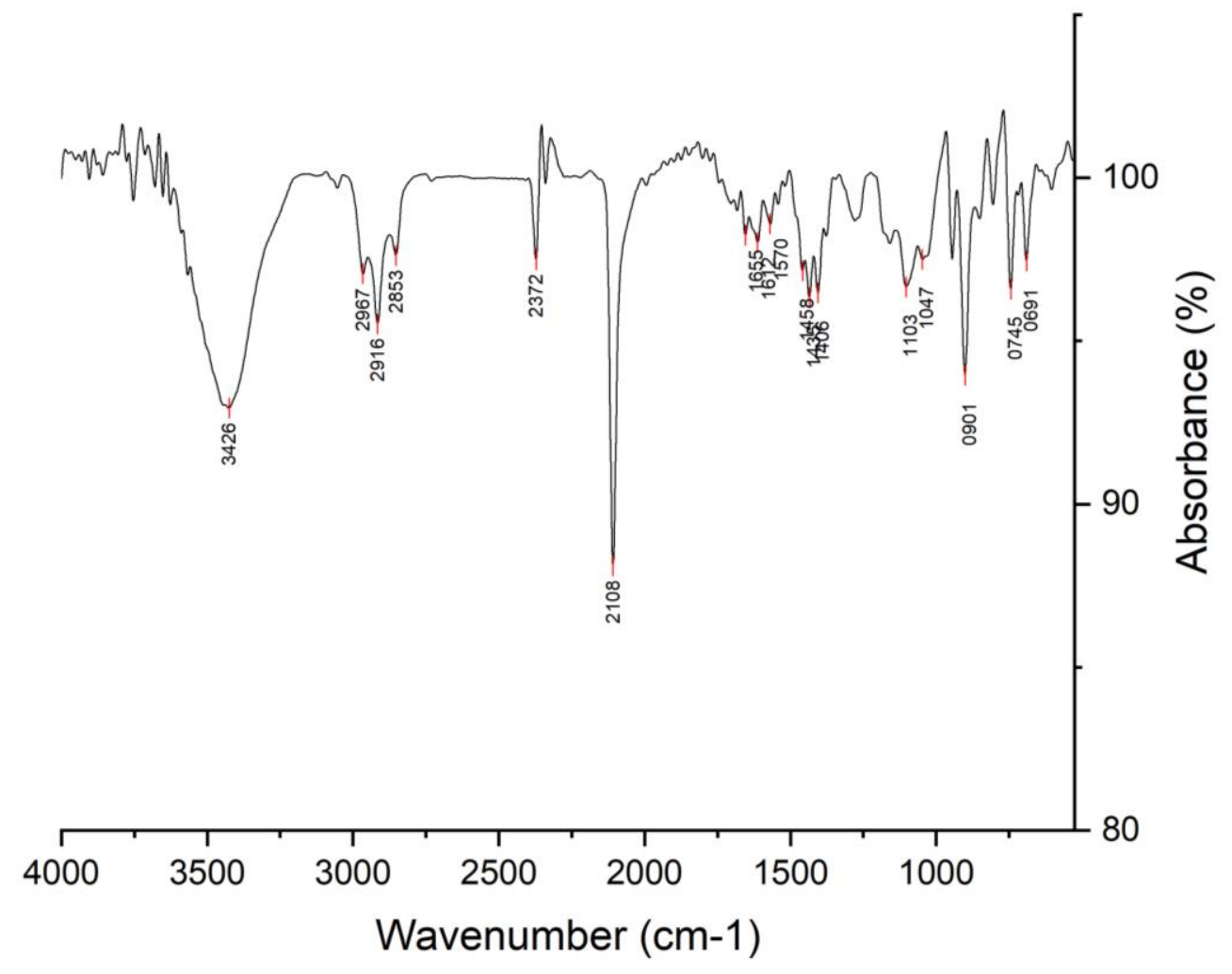

Figure S25. FT-IR spectrum of $c i s-\left[\mathrm{TcNCl}_{2}\left(\mathrm{CNAr}^{\mathrm{Mes} 2}\right)_{2}(\mathrm{MeOH})\right]$.

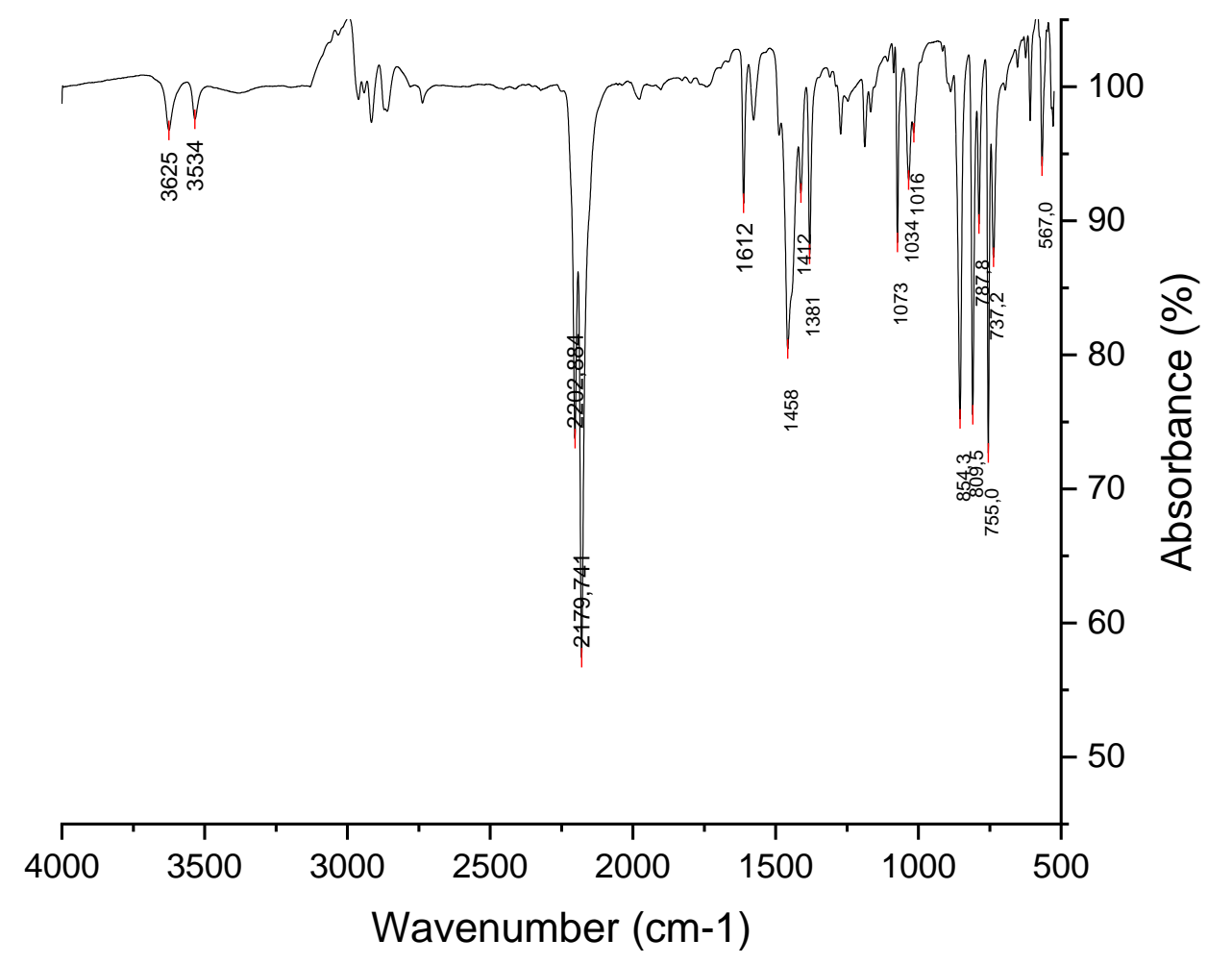


Figure S26. IR spectrum of cis-[ $\left[\mathrm{TcNCl}_{2}\left(\mathrm{CNArMes}_{2}\right)_{2}\left(\mathrm{H}_{2} \mathrm{O}\right)\right]$.

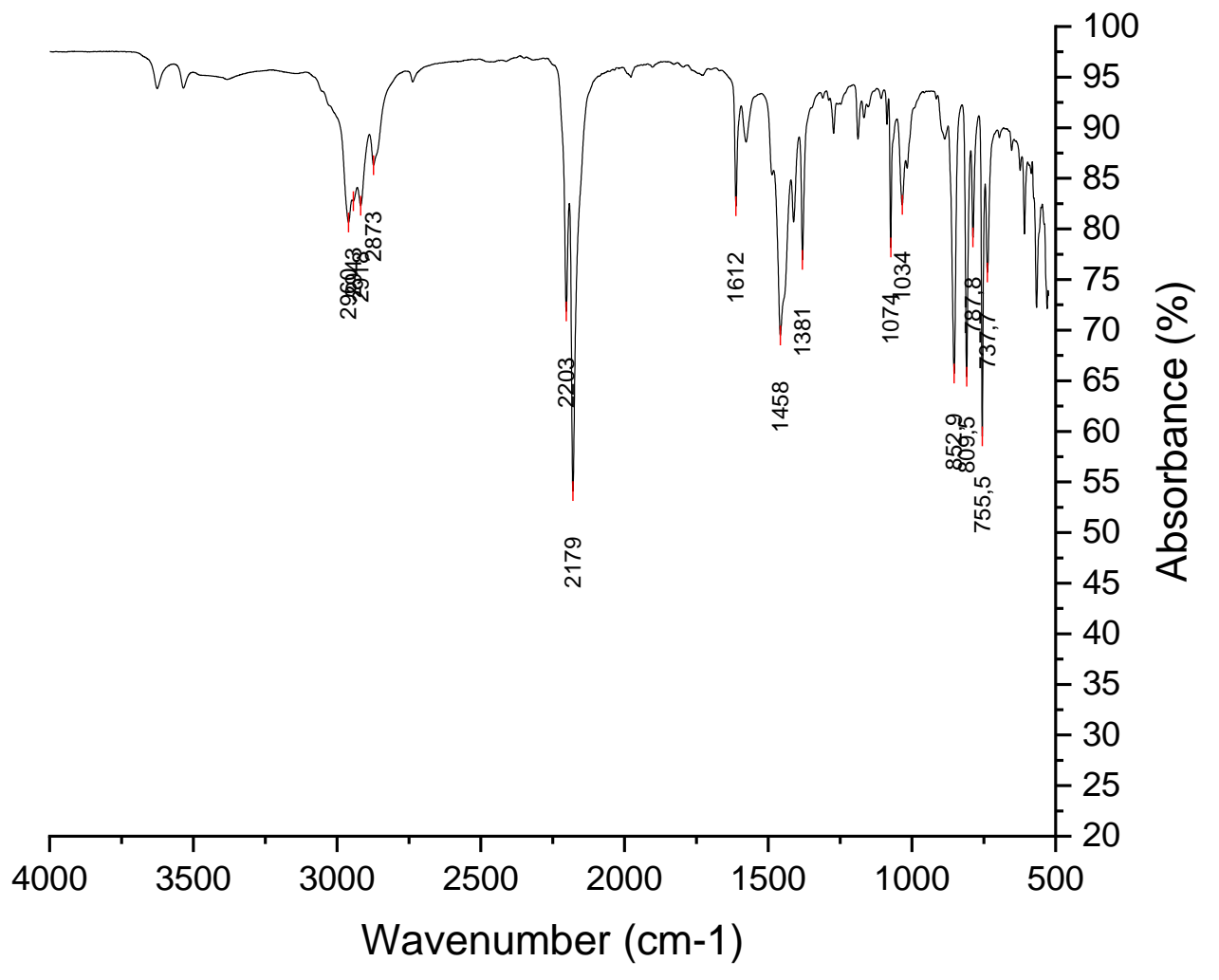

Figure S27. $1 \mathrm{H}$ NMR spectrum of $c i s-\left[\operatorname{TcNCl}_{2}\left(\mathrm{CNAr}^{\mathrm{Mes} 2}\right)_{2}\left(\mathrm{H}_{2} \mathrm{O}\right)\right]$.

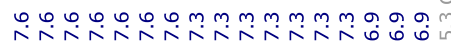

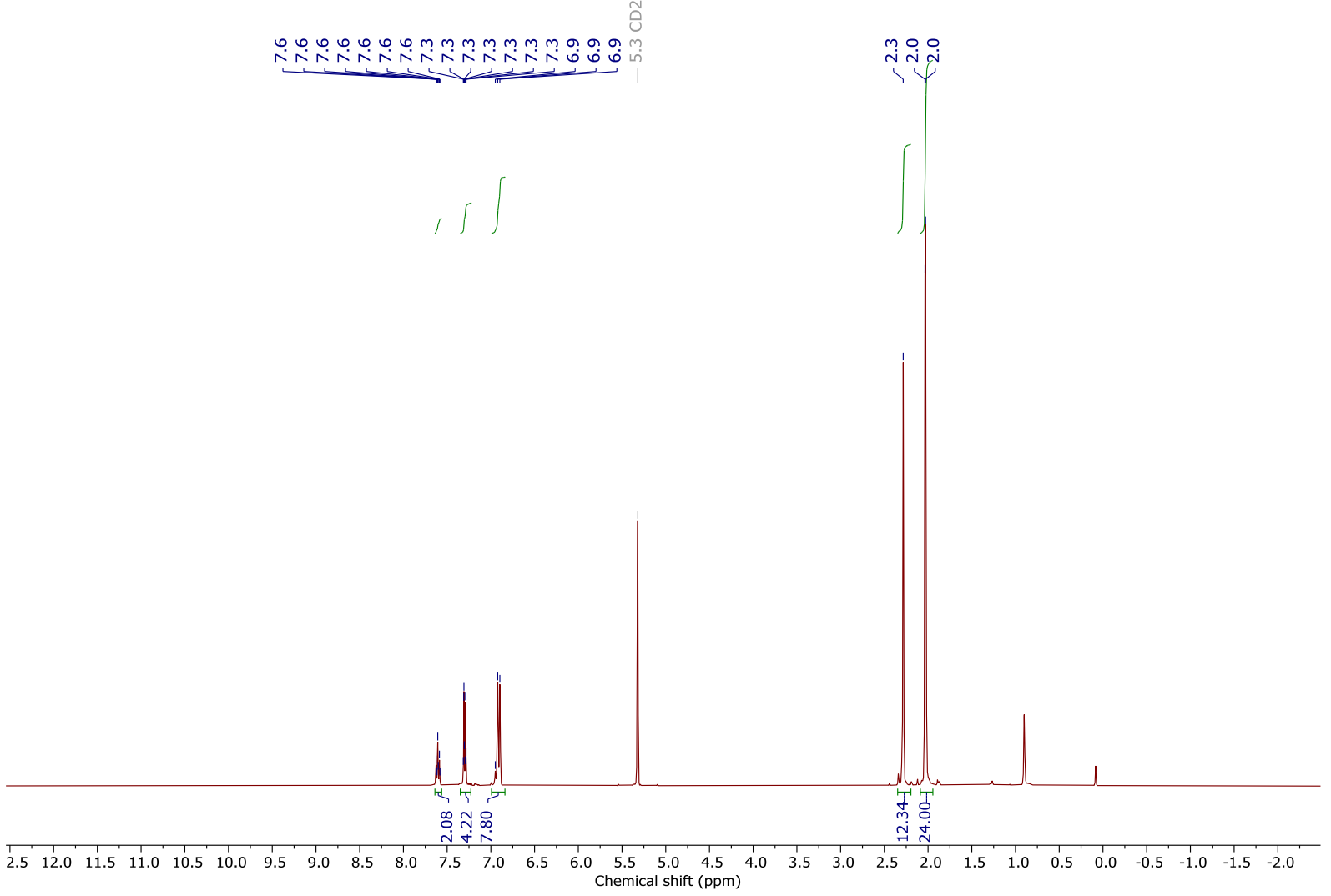


Figure S28. ${ }^{1} \mathrm{H}$ NMR spectrum of $c i s-\left[\mathrm{TcNBr}_{2}\left(\mathrm{CNAr}^{\mathrm{Mes} 2}\right)_{2}\right]$.

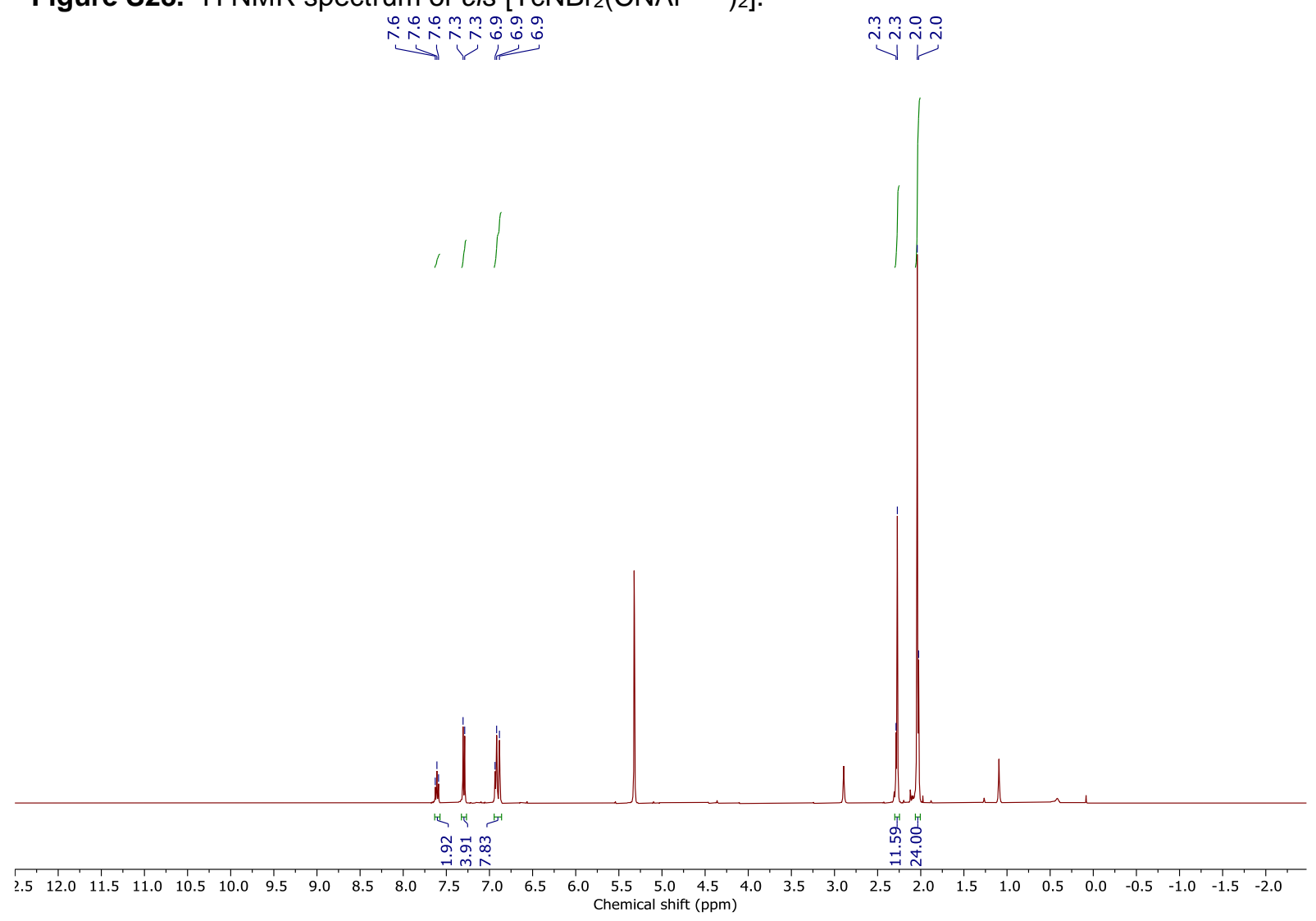

Figure S29. FT-IR spectrum of $c i s-\left[\mathrm{TcNBr}_{2}\left(\mathrm{CNAr}^{\mathrm{Mes} 2}\right)_{2}\right]$.

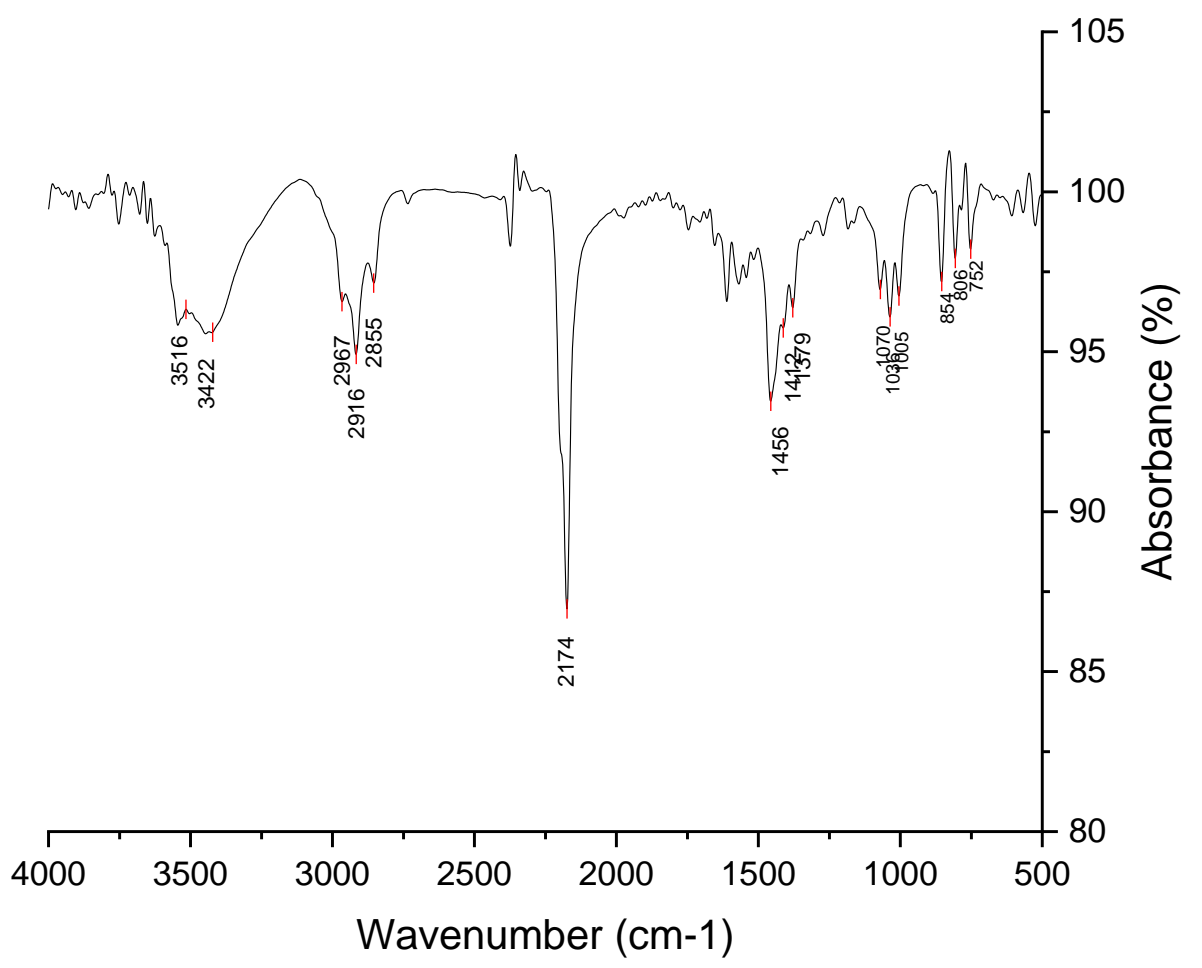

